\title{
PURE POINT SPECTRUM OF THE FLOQUET HAMILTONIAN FOR THE QUANTUM HARMONIC OSCILLATOR UNDER TIME QUASI- PERIODIC PERTURBATIONS
}

\begin{abstract}
W.-M. WANG*
Abstract. We prove that the $1-d$ quantum harmonic oscillator is stable under spatially localized, time quasi-periodic perturbations on a set of Diophantine frequencies of positive measure. This proves a conjecture raised by Enss-Veselic in their 1983 paper [EV] in the general quasi-periodic setting. The motivation of the present paper also comes from construction of quasi-periodic solutions for the corresponding nonlinear equation.
\end{abstract}

\section{Contents}

1. Introduction and statement of the theorem

2. The Floquet Hamiltonian in the Hermite-Fourier basis

3. Exponential decay of Green's functions at fixed $E$ : estimates in $\theta$

4. Frequency estimates and the elimination of $E$

5. Proof of the Theorem

I am deeply indebted to the referee for a thorough reading of the paper and for many helpful comments.

*Partially supported by NSF grant DMS-05-03563

Typeset by $\mathcal{A} \mathcal{M} \mathcal{S}-\mathrm{T}_{\mathrm{E}} \mathrm{X}$ 


\section{Introduction and statement of the theorem}

The stability of the quantum harmonic oscillator is a long standing problem since the establishment of quantum mechanics. The Schrödinger equation for the harmonic oscillator in $\mathbb{R}^{n}$ (in appropriate coordinates) is the following:

$$
-i \frac{\partial}{\partial t} \psi=\frac{1}{2} \sum_{i=1}^{n}\left(-\frac{\partial^{2}}{\partial x_{i}^{2}}+x_{i}^{2}\right) \psi
$$

where we assume

$$
\psi \in C^{1}\left(\mathbb{R}, L^{2}\left(\mathbb{R}^{n}\right)\right)
$$

for the moment. We start from the 1 dimensional case, $n=1$. (1.1) then reduces to

$$
-i \frac{\partial}{\partial t} \psi=\frac{1}{2}\left(-\frac{\partial^{2}}{\partial x^{2}}+x^{2}\right) \psi
$$

The Schrödinger operator

$$
H \stackrel{\text { def }}{=}\left(-\frac{d^{2}}{d x^{2}}+x^{2}\right)
$$

is the 1-d harmonic oscillator. Since $H$ is independent of $t$, it is amenable to a spectral analysis. It is well known that $H$ has pure point spectrum with eigenvalues

$$
\lambda_{n}=2 n+1, \quad n=0,1 \ldots
$$

and eigenfunctions (the Hermite functions)

$$
h_{n}(x)=\frac{H_{n}(x)}{\sqrt{2^{n} n !}} e^{-x^{2} / 2}, \quad n=0,1 \ldots
$$

where $H_{n}(x)$ is the $n^{\text {th }}$ Hermite polynomial, relative to the weight $e^{-x^{2}}\left(H_{0}(x)=1\right)$ and

$$
\begin{aligned}
& \int_{-\infty}^{\infty} e^{-x^{2}} H_{m}(x) H_{n}(x) d x \\
= & 2^{n} n ! \sqrt{\pi} \delta_{m n} .
\end{aligned}
$$

Using (1.4-1.6), the normalized $L^{2}$ solutions to (1.1) are all of the form

$$
\psi(x, t)=\sum_{n=0}^{\infty} a_{n} h_{n}(x) e^{i \frac{\lambda_{n}}{2} t} \quad\left(\sum\left|a_{n}\right|^{2}=1\right)
$$

corresponding to the initial condition

$$
\psi(x, 0)=\sum_{n=0}^{\infty} a_{n} h_{n}(x) \quad\left(\sum\left|a_{n}\right|^{2}=1\right) .
$$

The functions in (1.7) are almost-periodic (in fact periodic here) in time with frequencies $\lambda_{n} / 4 \pi, n=0,1 \ldots$ 
Equation (1.2) generates a unitary propagator $U(t, s)=U(t-s, 0)$ on $L^{2}(\mathbb{R})$. Since the spectrum of $H$ is pure point, $\forall u \in L^{2}(\mathbb{R}), \forall \epsilon, \exists R$, such that

$$
\inf _{t}\|U(t, 0) u\|_{L^{2}(|x| \leq R)} \geq(1-\epsilon)\|u\|
$$

by using eigenfunction (Hermite function) expansions.

The harmonic oscillator (1.3) is an integrable system. The above results are classical. It is natural to ask how much of the above picture remains under perturbation, when the system is no longer integrable. In this paper, we investigate stability of the 1-d harmonic oscillator under time quasi-periodic, spatially localized perturbations. To simplify the exposition, we study the following "model" equation:

$$
-i \frac{\partial}{\partial t} \psi=\frac{1}{2}\left(-\frac{\partial^{2}}{\partial x^{2}}+x^{2}\right) \psi+\delta\left|h_{0}(x)\right|^{2} \sum_{k=1}^{\nu} \cos \left(\omega_{k} t+\phi_{k}\right) \psi
$$

on $C^{1}\left(\mathbb{R}, L^{2}(\mathbb{R})\right)$, where

$$
\begin{aligned}
& 0<\delta \ll 1 \\
& \omega=\left\{\omega_{k}\right\}_{k=1}^{\nu} \in[0,2 \pi)^{\nu}, \\
& \phi=\left\{\phi_{k}\right\}_{k=1}^{\nu} \in[0,2 \pi)^{\nu}, \\
& h_{0}(x)=e^{-x^{2} / 2} \text { is the 0th Hermite function. }
\end{aligned}
$$

In particular, we shall study the validity of (1.9) for solutions to (1.10), when $U$ is the propagator for (1.10). The method used here can be generalized to treat the equation

$$
-i \frac{\partial}{\partial t} \psi=\frac{1}{2}\left(-\frac{\partial^{2}}{\partial x^{2}}+x^{2}\right) \psi+\delta V(t, x)
$$

where $V$ is $C_{0}^{\infty}$ in $x$ and analytic, quasi-periodic in $t$.

The perturbation term, $\mathcal{O}(\delta)$ term in $(1.10)$ is motivated by the nonlinear equation:

$$
-i \frac{\partial}{\partial t} \psi=\frac{1}{2}\left(-\frac{\partial^{2}}{\partial x^{2}}+x^{2}\right) \psi+M \psi+\delta|\psi|^{2} \psi \quad(0<\delta \ll 1),
$$

where $M$ is a Hermite multiplier, i.e., in the Hermite function basis,

$$
\begin{aligned}
M & =\operatorname{diag}\left(M_{n}\right), \quad M_{n} \in \mathbb{R}, \\
M u & =\sum_{n=0}^{\infty} M_{n}\left(h_{n}, u\right) h_{n}, \text { for all } u \in L^{2}(\mathbb{R}) .
\end{aligned}
$$

Specifically, (1.10) is motivated by the construction of time quasi-periodic solutions to (1.12) for appropriate initial conditions such as

$$
\psi(x, 0)=\sum_{i=1}^{\nu} c_{k_{i}} h_{k_{i}}(x)
$$


In (1.10), for computational simplicity, we take the spatial dependence to be $\left|h_{0}(x)\right|^{2}$ as it already captures the essence of the perturbation in view of $(1.12,1.13,1.5)$. The various computations and the Theorem extend immediately to more general finite combinations of $h_{k}(x)$.

\section{The Floquet Hamiltonian and formulation of stability}

It follows from $[Y 2,3]$ that $(1.10)$ generates a unique unitary propagator $U(t, s)$, $t, s \in \mathbb{R}$ on $L^{2}(\mathbb{R})$, so that for every $s \in \mathbb{R}$ and

$$
\begin{gathered}
u_{0} \in B^{2}=\left\{f \in L^{2}(\mathbb{R}) \mid\|f\|_{B^{2}}^{2}=\sum_{|\alpha+\beta| \leq 2}\left\|x^{\alpha} \partial_{x}^{\beta} f\right\|_{L^{2}}^{2}<\infty\right\}, \\
u(\cdot)=U(\cdot, s) u_{0} \in C^{1}\left(\mathbb{R}, L^{2}(\mathbb{R})\right) \cap C^{0}\left(\mathbb{R}, B^{2}\right)
\end{gathered}
$$

is a unique solution of $(1.10)$ in $L^{2}(\mathbb{R})$ satisfying $u(s)=u_{0}$.

When $\nu=1,(1.10)$ is time periodic with period $T=2 \pi / \omega$. The 1-period propagator $U(T+s, s)$ is called the Floquet operator. The long time behavior of the solutions to (1.10) can be characterized by means of the spectral properties of $U(T+s, s)$ $[\mathrm{EV}, \mathrm{Ho}, \mathrm{YK}]$. Furthermore the nature of the spectrum of $U$ is the same (apart from multiplicity) as that of the Floquet Hamiltonian $K[\mathrm{Y} 1]$ :

$$
K=i \omega \frac{\partial}{\partial \phi}+\frac{1}{2}\left(-\frac{\partial^{2}}{\partial x^{2}}+x^{2}\right) \psi+\delta\left|h_{0}(x)\right|^{2} \cos \phi
$$

on $L^{2}(\mathbb{R}) \otimes L^{2}(\mathbb{T})$, where $L^{2}(\mathbb{T})$ is $L^{2}[0,2 \pi)$ with periodic boundary conditions.

Decompose $L^{2}(\mathbb{R})$ into the pure point $\mathcal{H}_{p p}$ and continuous $\mathcal{H}_{c}$ spectral subspaces of the Floquet operator $U(T+s, s)$ :

$$
L^{2}(\mathbb{R})=\mathcal{H}_{p p} \oplus \mathcal{H}_{c}
$$

We have the following equivalence relations [EV,YK]: $u \in \mathcal{H}_{p p}(U(T+s, s))$ if and only if $\forall \epsilon>0, \exists R>0$, such that

$$
\inf _{t}\|U(t, s) u\|_{L^{2}(|x| \leq R)} \geq(1-\epsilon)\|u\|
$$

and $u \in \mathcal{H}_{c}(U(T+s, s))$ if and only if $\forall R>0$,

$$
\lim _{t \rightarrow \pm \infty} \frac{1}{t} \int_{0}^{t} d t^{\prime}\left\|U\left(t^{\prime}, s\right) u\right\|_{L^{2}(|x| \leq R)}^{2}=0
$$

(Needless to say, the above statements hold for general time periodic Schrödinger equations.)

When $\nu \geq 2,(1.10)$ is time quasi-periodic. The above constructions extend for small $\delta$, cf. [Be, E, JL] leading to the Floquet Hamiltonian $K$ :

$$
K=i \sum_{k=1}^{\nu} \omega_{k} \frac{\partial}{\partial \phi_{k}}+\frac{1}{2}\left(-\frac{\partial^{2}}{\partial x^{2}}+x^{2}\right) \psi+\delta\left|h_{0}(x)\right|^{2} \sum_{k=1}^{\nu} \cos \phi_{k}
$$


on $L^{2}(\mathbb{R}) \otimes L^{2}\left(\mathbb{T}^{\nu}\right)$, cf. [BW1]. This is related to the so called reducibility of skew product flows in dynamical systems, cf. [E]. We note that the Hermite-Fourier functions:

$$
e^{-i n \cdot \phi} h_{j}(x), \quad n \in \mathbb{Z}^{\nu}, \quad \phi \in \mathbb{T}^{\nu}, \quad j \in\{0,1 \ldots\}
$$

provide a basis for $L^{2}(\mathbb{R}) \otimes L^{2}\left(\mathbb{T}^{\nu}\right)$.

We say that the harmonic oscillator $H$ is stable if $K$ has pure point spectrum. Let $s \in \mathbb{R}$. This implies (by expansion using eigenfunctions of $K$ ) that given any $u \in L^{2}(\mathbb{R}), \forall \epsilon>0, \exists R>0$, such that

$$
\inf _{t}\|U(t, s) u\|_{L^{2}(|x| \leq R)} \geq(1-\epsilon)\|u\| \text {, a.e. } \phi,
$$

cf. [BW1, JL]. So (1.9) remains valid and we have dynamical stability.

We now state the main results pertaining to (1.10).

Theorem. There exists $\delta_{0}>0$, such that for all $0<\delta<\delta_{0}$, there exists $\Omega \subset[0,2 \pi)^{\nu}$ of positive measure, asymptotically full measure:

$$
\text { mes } \Omega \rightarrow(2 \pi)^{\nu} \quad \text { as } \delta \rightarrow 0 \text {, }
$$

such that for all $\omega \in \Omega$, the Floquet Hamiltonian $K$ defined in (1.15) has pure point spectrum: $\sigma(K)=\sigma_{\mathrm{pp}}$. Moreover the Fourier-Hermite coefficients of the eigenfunctions of $K$ have subexponential decay.

As an immediate consequence, we have

Corollary. Assume that $\Omega$ is as in the Theorem. Let $s \in \mathbb{R}$. For all $\omega \in \Omega$, all $u \in L^{2}(\mathbb{R})$, all $\epsilon>0$, there exists $R>0$, such that

$$
\inf _{t}\|U(t, s, \phi) u\|_{L^{2}(|x| \leq R)} \geq(1-\epsilon)\|u\| \text {, a.e. } \phi,
$$

where $U$ is the unitary propagator for (1.10).

We note that this good set $\Omega$ of $\omega$ is a subset of Diophantine frequencies. This is typical for KAM type of persistence theorem. Stability under time quasi-periodic perturbations as in (1.10) is, generally speaking a precursor for stability under nonlinear perturbation as in (1.12) (cf. [BW1,2]), where $M$ plays the role of $\omega$ and varies the tangential frequencies. The above Theorem resolves the Enss-Veselic conjecture dated from their 1983 paper [EV] in a general quasi-periodic setting.

A sketch of the proof of the Theorem.

Instead of working with $K$ defined on $L^{2}(\mathbb{R}) \otimes L^{2}\left(\mathbb{T}^{\nu}\right)$ directly, it is more convenient to work with its unitary equivalent $H$ on $\ell^{2}\left(\mathbb{Z}^{\nu} \times\{0,1 \ldots\}\right)$, using the Hermite-Fourier basis in (1.16). We have

$$
H=\operatorname{diag}\left(n \cdot \omega+j+\frac{1}{2}\right)+\frac{\delta}{2} W \otimes \Delta
$$


on $\ell^{2}\left(\mathbb{Z}^{\nu} \times\{0,1 \ldots\}\right)$, where $W$ acts on the $j$ indices, $j=0,1,2 \ldots$,

$$
W_{j j^{\prime}} \sim \frac{1}{\sqrt{j+j^{\prime}}} e^{-\frac{\left(j-j^{\prime}\right)^{2}}{2\left(j+j^{\prime}\right)}} \quad \text { for } j+j^{\prime} \gg 1
$$

$\Delta$ acts on the $n$ indices, $n \in \mathbb{Z}^{\nu}$,

$$
\begin{aligned}
\Delta_{n n^{\prime}} & =1, & & \left|n-n^{\prime}\right|_{\ell^{1}}=1, \\
& =0, & & \text { otherwise. }
\end{aligned}
$$

The reduction from (1.15) to (1.19-1.21) is performed in section 2. The main work is to compute $W$, which involves integrals of products of Hermite functions. We will explain shortly this computation, which is independent from the main thread of construction.

The principal new feature here is that $W$ is long range. The $j^{\text {th }}$ row has width $\mathcal{O}(\sqrt{j})$ about the diagonal element $W_{j j}$. It is not and cannot be approximated by a convolution matrix. The potential $x^{2}$ breaks translational invariance. The annihilation and creation operators of the harmonic oscillator $a=\frac{1}{\sqrt{2}}\left(\frac{d}{d x}+x\right), a^{*}=\frac{1}{\sqrt{2}}\left(-\frac{d}{d x}+x\right)$, satisfying $\left[a, a^{*}\right]=1$, are generators of the Heisenberg group. So (1.19) presents a new class of problems distinct from that considered in [B1-3, BW1, 2, EK, Ku1, KP].

The proof of pure point spectrum of $H$ is via proving pointwise decay as $|x-y| \rightarrow \infty$ of the finite volume Green's functions: $\left(H_{\Lambda}-E\right)^{-1}(x, y)$, where $\Lambda$ are finite subsets of $\mathbb{Z}^{\nu} \times\{0,1 \ldots\}$ and $\Lambda \nearrow \mathbb{Z}^{\nu} \times\{0,1 \ldots\}$. We need decay of the Green's functions at all scales, as assuming $E$ an eigenvalue, a priori we do not have information on the center and support of its eigenfunction $\psi$. The regions $\Lambda$ where $\left(H_{\Lambda}-E\right)^{-1}$ has pointwise decay is precisely where we establish later that $\psi$ is small there.

For the initial scales, the estimates on $G_{\Lambda}(E)=\left(H_{\Lambda}-E\right)^{-1}$ are obtained by direct perturbation theory in $\delta$ for $0<\delta \ll 1$. For subsequent scales, the proof is a multiscale induction process using the resolvent equation. Assume we have estimates on $G_{\Lambda^{\prime}}$ for cubes $\Lambda^{\prime}$ at scale $L^{\prime}$ and $\Lambda$ is a cube at a larger scale $L, L \gg L^{\prime}$. Intuitively, if we could establish that for most of $\Lambda^{\prime} \subset \Lambda, G_{\Lambda^{\prime}}(E)$ has pointwise decay, then assuming we have some a priori estimates on $G_{\Lambda}(E)$, we should be able to prove that $G_{\Lambda}(E)$ also has pointwise decay.

There are "two" directions in the problem, the higher harmonics direction $n$ and the spatial direction $j$. The off-diagonal part of $H$ is Toeplitz in the $n$ direction, corresponding to the discrete Laplacian $\Delta$. Since the frequency $\omega$ is in general a vector (if $\nu \geq 2$ ), $n \cdot \omega$ does not necessarily $\rightarrow \infty$ as $|n| \rightarrow \infty$. So the $n$ direction is non perturbative. We use estimates on $G_{\Lambda^{\prime}}$ and semi-algebraic techniques as in [BGS,BW1] to control the number of resonant $\Lambda^{\prime}$, where $G_{\Lambda^{\prime}}$ is large, in $\Lambda$.

In the $j$ direction, we do analysis, i.e., perturbation theory. This is the new feature. From (1.19) and Schur's lemma, $\|W \otimes \Delta\|=\mathcal{O}(1)$. So the $\ell^{2}$ norm of the perturbation does not decay (relative to eigenvalue spacing) in $j$. However when $\delta=0, H$ is diagonal with eigenvalues $n \cdot \omega+j$ and eigenfunctions $\delta_{n, j}$, the canonical basis for $\ell^{2}\left(\mathbb{Z}^{\nu} \times\{0,1 \ldots\}\right)$. We have

$$
\left\|[W \otimes \Delta] \delta_{n, j}\right\|=\mathcal{O}\left(\frac{1}{j^{1 / 4}}\right) \quad(j \geq 1),
$$


which decays in $j$.

This is intuitively reasonable, as $W$ stems from a spatially localized perturbation from (1.10). As $j$ increases, The Hermite functions $h_{j}$ become more extended, c.f. (1.5). So the effect of the spatial perturbation should decrease as $j$ increases.

Assuming $\omega$ is Diophantine:

$$
\|n \cdot \omega\|_{\mathbb{T}} \geq \frac{c}{|n|^{\alpha}} \quad(c>0, n \neq 0, \alpha>2 \nu),
$$

where $\|\cdot\|_{\mathbb{T}}$ is the distance to the nearest integer, this enables us to preserve local eigenvalue spacing for $\Lambda$ which are appropriately proportioned in $n, j$. This in turn leads to decay of Green's functions. Combining the estimates in the $n$ and $j$ directions, we obtain estimates on the Green's function at the larger scale $L$.

Integrals of products of Hermite functions.

From $(1.15,1.19)$, computation of $W$ involves computing the following integrals:

$$
\begin{aligned}
& \int_{-\infty}^{\infty} h_{0}^{2}(x) h_{m}(x) h_{n}(x) d x \\
= & \frac{1}{\sqrt{2^{n+m} m ! n !}} \int_{-\infty}^{\infty} e^{-2 x^{2}} H_{0}^{2}(x) H_{m}(x) H_{n}(x) d x, \quad m, n=0,1 \ldots
\end{aligned}
$$

where $H_{m}, H_{n}$ are respectively the $m^{\text {th }}, n^{\text {th }}$ Hermite polynomial, $H_{0}(x)=1$.

Let

$$
I=\int_{-\infty}^{\infty} e^{-2 x^{2}} H_{0}^{2}(x) H_{m}(x) H_{n}(x) d x .
$$

The idea is to view $e^{-x^{2}} H_{0}^{2}(x)$ as $e^{-x^{2}} H_{0}(\sqrt{2} x)$, i.e., the $0^{\text {th }}$ Hermite function relative to the weight $e^{-2 x^{2}}$ and to use the generating function of Hermite polynomials to reexpress

$$
H_{m}(x) H_{n}(x)=\sum_{\ell=0}^{m+n} a_{\ell} H_{\ell}(\sqrt{2} x) .
$$

We then have

$$
\begin{aligned}
I & =a_{0} \int\left[H_{0}(\sqrt{2} x)\right]^{2} e^{-2 x^{2}} d x \\
& =a_{0} \sqrt{\pi / 2}
\end{aligned}
$$

using (1.6). This computation is carried out in section 2, (2.7-2.10), recovering an apparently classical result, which could be found in e.g., [GR, PBM].

More generally, we are interested in computing

$$
I=\int_{-\infty}^{\infty} e^{-2 x^{2}} H_{p}(x) H_{q}(x) H_{m}(x) H_{n}(x) d x, \quad p, q, m, n=0,1 \ldots
$$


which are needed for the nonlinear equation or if we consider more general perturbations of the harmonic oscillator. Following the same line of arguments, we decompose $H_{p}(x) H_{q}(x)$ into

$$
H_{p}(x) H_{q}(x)=\sum_{\ell=0}^{p+q} b_{\ell} H_{\ell}(\sqrt{2} x) .
$$

Combining (1.23) with (1.25), and assuming (without loss of generality), $p+q \leq m+n$, we then have

$$
I=\sum_{\ell=0}^{p+q} a_{\ell} b_{\ell} c_{\ell}
$$

where $c_{\ell}=\int_{-\infty}^{\infty}\left[H_{\ell}(\sqrt{2} x)\right]^{2} e^{-2 x^{2}} d x$.

The computation for general $p, q$ is technically more involved and is carried out in [W]. Unlike the special case $p=q=0$, we did not find the corresponding result for general $p, q$ in existing literature.

The computation of $I$ in (1.24) is exact (see (2.10)), reflecting the integrable nature of the quantum harmonic oscillator. The proof of the theorem is, however, general. It is applicable as soon as the kernel $W$ satisfies (1.20). Following the precedent discussion on $I$ for general $p, q$, and using properties of the Hermite series (cf. [T] and references therein), one should be able to extend the Theorem to $V$, which are $C_{0}^{\infty}$ in $x$ and analytic quasi-periodic in $t$, leading to perturbation kernels in the Hermite-Fourier basis satisfying conditions similar to (1.20) in the $j$ direction and exponential decay condition in the $n$ direction.

When the perturbation $V$ is independent of time and is a $0^{\text {th }}$ order symbol, satisfying

$$
\left|\partial^{\alpha} V\right| \leq C_{\alpha}(1+|x|)^{-\alpha}, \quad \alpha=0,1 \ldots
$$

the corresponding Schrödinger equation has been studied in e.g., [BBL, KRY, Z], where it was shown that certain properties of the harmonic oscillator equation extend to the perturbed equation. The spectral property needed for the construction here is more detailed and stringent. Hence it is reasonable to believe that the set of potentials $V$ will be more restrictive than that in (1.26)

\section{Some perspectives on the Theorem}

The Theorem shows that for small $\delta$, there is a subset $\Omega \subset[0,2 \pi)^{\nu}$ of Diophantine frequencies of positive measure, such that if $\omega \in \Omega$, then (1.9) is satisfied. Hence spatially localized solutions remain localized for all time. It is natural to ask what happens if the forcing frequencies $\omega$ are in the complement set, $\omega \in \Omega^{c}$.

If $\omega$ is rational, the perturbing potential $V$ is bounded and has sufficiently fast decay at infinity, it is known from general compactness argument [EV] that the Floquet Hamiltonian has pure point spectrum. In our example, this can be seen as follows. In (1.10) restricting to periodic perturbation $(\nu=1)$, it is easy to see that for

$$
\forall \omega, A=(n \omega+j+z)^{-1} W \otimes \Delta, \text { where } \Im z=1 \text { is compact. }
$$

Assume $\omega$ is rational: $\omega=p / q,(q \neq 0)$. Since $H_{0}=n \omega+j$ has pure point spectrum (with infinite degeneracy) and the spacing between different eigenvalues is $1 / q,(1.27)$ 
implies that $H$ has pure point spectrum. When $\omega$ is irrational, $H_{0}$ typically has dense spectrum. No conclusion can be drawn from (1.27).

It is worth remarking that (1.27) holds for all scalar $\omega$. In the quasi-periodic case, $\omega$ is a vector, the compactness argument breaks down. The proof of Lemma 3.5 in the present paper is a replacement.

If $V$ is unbounded, we have a different situation. The results in [HLS, GY] combined with $[\mathrm{YK}]$ show that for the following unbouded time periodically perturbed harmonic oscillator:

$$
i \frac{\partial u}{\partial t}=\frac{1}{2}\left(-\Delta+x^{2}\right) u+2 \epsilon(\sin t) x_{1} u+\mu V(t, x) u, \quad x=\left(x_{1}, \ldots x_{n}\right) \in \mathbb{R}^{n}
$$

where $V(t, x)$ is a real valued smooth function of $(t, x)$, satisfying

$$
V(t+2 \pi, x)=V(t), \quad|V(t, x)| \lesssim|x| \quad \text { as } x \rightarrow \infty, \quad\left|\partial_{x}^{\alpha} V(t, x)\right| \leq C_{\alpha}, \quad|\alpha| \geq 1
$$

the solutions diffuse to infinity as $t \rightarrow \infty$. More precisely, for all $u_{0} \in L^{2}\left(\mathbb{R}^{n}\right) \cap H^{2}\left(\mathbb{R}^{n}\right)$, for any $R>0$, the solution $u_{t}$ satisfies

$$
\lim _{T \rightarrow \pm \infty} \frac{1}{T} \int_{0}^{T} d t\left\|u_{t}\right\|_{L^{2}(|x| \leq R)}=0
$$

In (1.28), $\nu=1$ (periodic), $\omega=1, \omega \in \Omega^{c},(1.29)$ is an opposite of (1.9). However the perturbation is unbounded. Moreover the proof in [GY] uses in an essential way that the potential is linear at infinity, hence positivity of the commutator: $\left[\frac{d}{d x_{1}}, x_{1}\right]=1$.

In the exactly solvable case where the time periodic perturbations is quadratic in the spatial coordinates, it is known that the Floquet Hamiltonian exhibits a transition between pure point and continuous spectrum as the frequency is varied [Co1]. The perturbation there is again unbounded.

Some related results.

To our knowledge, when $\omega \in \Omega$ is nonresonant, there were no results in the literature on the perturbed harmonic oscillator equation of type (1.10), even in the time periodic case, i.e., $\omega \in[0,2 \pi)$. The main difficulties encountered by the traditional KAM method seem to be (i) the eigenvalue spacing for the unperturbed operator does not grow, $\lambda_{k+1}-\lambda_{k}=1$, (ii) the perturbation $W$ in the Hermite basis has slow decay $(1.20)$.

When the eigenvalue spacing for the unperturbed operator grows: $\left|E_{j+1}-E_{j}\right|>$ $j^{\beta} \quad(\beta>0)$, which corresponds to a potential growing faster than quadratically at infinity, and when the perturbation is periodic in time, related stability results were proven in [DS]. In [Co2], under time periodic perturbation and replacing $W$ in (1.20) by a faster decaying kernel, hence decaying norm in $j$, which no longer corresponds to the physical case of harmonic oscillator under time periodic, spatially localized perturbation, stability results were also proven. Both papers used some modified KAM method. 


\section{Motivation for studying (1.10)}

As mentioned earlier, the motivation for analyzing (1.10) partly comes from the nonlinear equation (1.12). In [B1-3, EK], time quasi-periodic solutions were constructed for the nonlinear Schrödinger equation in $\mathbb{R}^{d}$ with Dirichlet or periodic boundary condition

$$
i \frac{\partial}{\partial t} \psi=(-\Delta+M) \psi+\delta|\psi|^{2 p} \psi, \quad\left(p \in \mathbb{N}^{+} ; 0<\delta \ll 1\right)
$$

where $M$ is a Fourier multiplier; see $[\mathrm{Ku} 1, \mathrm{KP}]$ for the Dirichlet case in $\mathbb{R}$ with a potential in place of $M$. In [BW2], time quasi-periodic solutions were constructed for the nonlinear random Schrödinger equation in $\mathbb{Z}^{d}$

$$
i \frac{\partial}{\partial t} \psi=(-\epsilon \Delta+V) \psi+\delta|\psi|^{2 p} u, \quad\left(p \in \mathbb{N}^{+} ; \epsilon, 0<\delta \ll 1\right),
$$

where $V=\left\{v_{j}\right\}_{j \in \mathbb{Z}^{d}}$ is a family of random variables.

The proofs in [B1-3, BW2] use operator method, which traces its origin to the study of Anderson localization [FS]. This method was first applied in the context of Hamiltonian PDE in [CW]. The proofs in [EK, Ku1, KP] use KAM type of method.

In (1.30) (specializing to 1-d), the eigenvalues of the linear operator are $n^{2}$, so $E_{n+1}-E_{n} \sim n$, the eigenfunctions $e^{i n x}$, however, are extended: $\left|e^{i n x}\right|=1$ for all $x$. Let us call this case $A$, where there is eigenvalue separation. In (1.31), the eigenvalues of the linear operator form a dense set, the eigenfunctions, on the other hand are not only localized but localized about different points in $\mathbb{Z}^{d}$ from Anderson localization theory, see e.g., [GB, GK]. This is case $B$, where there is eigenfunction separation. The existence of time quasi-periodic solutions, i.e., KAM type of solutions in $A$ is a consequence of eigenvalue separation; while in $B$, eigenfunction separation.

Equation (1.10) and its nonlinear counterpart

$$
-i \frac{\partial}{\partial t} \psi=\frac{1}{2}\left(-\frac{\partial^{2}}{\partial x^{2}}+x^{2}\right) \psi+M \psi+\delta|\psi|^{2 p} \psi, \quad\left(p \in \mathbb{N}^{+} ; 0<\delta \ll 1\right),
$$

where $M$ is a Hermite multiplier, stand apart from both $(1.30,1.31)$. It is neither $A$, nor $B$. There is eigenvalue spacing, but it is a constant: $\lambda_{n+1}-\lambda_{n}=1$. In particular, it does not grow with $n$. The eigenfunctions (Hermite functions) $h_{n}$ are "localized" about the origin. But they become more extended as $n$ increases because of the presence of the Hermite polynomials, cf. (1.5). This in turn leads to the long range kernel $W$ in (1.20) and long range nonlinearity in (1.32) in the Hermite function basis, cf. [W].

From the KAM perspective a la Kuksin, this is a borderline case, where Theorem 1.1 in [Ku2] does not apply. The more recent KAM type of theorem in [EK] does not apply either, because $W$ is long range and not close to a Toeplitz matrix (cf.(1.20)) for the reasons stated earlier. These are the features which make $(1.10,1.32)$ interesting from a mathematics point of view, aside from its apparent relevance to physics. 


\section{The Floquet Hamiltonian in the Hermite-Fourier basis}

Recall from section 1, the Floquet Hamiltonian

$$
K=i \sum_{k=1}^{\nu} \omega_{k} \frac{\partial}{\partial \phi_{k}}+\frac{1}{2}\left(-\frac{\partial^{2}}{\partial x^{2}}+x^{2}\right)+\delta\left|h_{0}(x)\right|^{2} \sum_{k=1}^{\nu} \cos \phi_{k}
$$

on $L^{2}(\mathbb{R}) \otimes L^{2}\left(\mathbb{T}^{\nu}\right)$, where

$$
\begin{aligned}
& 0<\delta \ll 1, \\
& \omega_{k} \in[0,2 \pi), \quad k=1, \ldots, \nu \\
& \phi_{k} \in[0,2 \pi), \quad k=1, \ldots, \nu \\
& h_{0}(x)=e^{-x^{2} / 2} .
\end{aligned}
$$

As mentioned in section $1, h_{0}(x)$ is the $0^{\text {th }}$ Hermite function, $0^{\text {th }}$ eigenfunction of the 1-d harmonic oscillator and more generally,

$$
\begin{aligned}
\left(-\frac{d^{2}}{d x^{2}}+x^{2}\right) h_{n} & =\lambda_{n} h_{n} \\
\lambda_{n} & =2 n+1, \quad n=0,1 \ldots \\
h_{n}(x) & =\frac{H_{n}(x)}{\sqrt{2^{n} n !}} e^{-x^{2} / 2}, \quad n=0,1 \ldots
\end{aligned}
$$

where $H_{n}(x)$ is the $n^{\text {th }}$ Hermite polynomial, relative to the weight $e^{-x^{2}}\left(H_{0}(x)=1\right)$ and

$$
\begin{aligned}
& \int_{-\infty}^{\infty} e^{-x^{2}} H_{m}(x) H_{n}(x) d x \\
= & 2^{n} n ! \sqrt{\pi} \delta_{m n}
\end{aligned}
$$

Integral of products of Hermite functions

We express (2.1) in the Hermite function basis for small $\delta$ and compute the integral:

$$
\begin{aligned}
& \int_{-\infty}^{\infty} h_{0}^{2}(x) h_{m}(x) h_{n}(x) d x \\
= & \frac{1}{\sqrt{2^{n+m} m ! n !}} \int_{-\infty}^{\infty} e^{-2 x^{2}} H_{0}^{2}(x) H_{m}(x) H_{n}(x) d x, \quad m, n=0,1 \ldots
\end{aligned}
$$

The integral of the more general product

$$
\begin{aligned}
& \int_{-\infty}^{\infty} e^{-2 x^{2}} H_{p}(x) H_{q}(x) H_{m}(x) H_{n}(x) d x \\
= & \left(H_{p}(x) H_{q}(x) e^{-x^{2}}, H_{m}(x) H_{n}(x) e^{-x^{2}}\right), \quad p, q, m, n=0,1 \ldots
\end{aligned}
$$

is done in $[\mathrm{W}]$. 
Since

We use generating functions of Hermite polynomials to find $a_{\ell}$ of (1.23) as follows.

$$
\begin{aligned}
e^{2 t x-t^{2}} & =\sum_{n=0}^{\infty} \frac{t^{n}}{n !} H_{n}(x), \\
e^{2 s x-s^{2}} & =\sum_{m=0}^{\infty} \frac{s^{m}}{m !} H_{m}(x),
\end{aligned}
$$

which can be found in any mathematics handbook (cf. [CFKS, T] for connections with the Mehler formula), multiplying $(2.7,2.8)$, we obtain

$$
\begin{aligned}
& e^{2(t+s) x-\left(t^{2}+s^{2}\right)}=\sum_{n, m} \frac{t^{n} s^{m}}{n ! m !} H_{n}(x) H_{m}(x) \\
= & e^{2\left(\frac{t+s}{\sqrt{2}}\right) \sqrt{2} x-\left(\frac{t+s}{\sqrt{2}}\right)^{2}} \cdot e^{-\frac{1}{2}(t-s)^{2}} \\
= & \sum_{\ell=0}^{\infty} H_{\ell}(\sqrt{2} x) \cdot \frac{\left(\frac{t+s}{\sqrt{2}}\right)^{\ell}}{\ell !} \cdot \sum_{p=0}^{\infty}(-1)^{p} \frac{(t-s)^{2 p}}{2^{p} p !} .
\end{aligned}
$$

From (1.23), we are only interested in the coefficient in front of $H_{0}(\sqrt{2} x)$. So we set $\ell=0$. To obtain $a_{0}$, we equate the coefficient in front of $t^{n} s^{m}$. Comparing the LHS with RHS of (2.9), $n, m$ must have the same parity, otherwise it is 0 . We deduce

$$
\begin{aligned}
a_{0} & =\frac{(-1)^{\frac{n-m}{2}}}{2^{\frac{m+n}{2}}\left(\frac{m+n}{2}\right) !} \cdot(n+m) !, & & n, m \text { same parity } \\
& =0 & & \text { otherwise, }
\end{aligned}
$$

by taking $2 p=n+m$, which is the only contributing term.

Taking into account the normalization factors in the third equation of (2.3), we then obtain

\section{Lemma 2.1.}

$$
\begin{array}{rlr}
W_{m n} \stackrel{\text { def }}{=} \int_{-\infty}^{\infty} h_{0}^{2}(x) h_{m}(x) h_{n}(x) d x & \\
& =\frac{(-1)^{\frac{n-m}{2}}}{2^{m+n} \sqrt{m ! n !}} \cdot \frac{(m+n) !}{\left(\frac{m+n}{2}\right) !} \sqrt{\frac{\pi}{2}} \quad \text { m, n same parity } \\
& =0 & \text { otherwise. }
\end{array}
$$

Let

$$
N=\frac{n+m}{2}, k=\frac{n-m}{2},
$$

assuming $n \geq m$, without loss. When $N \gg 1$,

$$
\begin{aligned}
W_{m n} & =\left[1+\mathcal{O}\left(\frac{1}{N}\right)\right] \frac{(-1)^{\frac{n-m}{2}} N !}{\sqrt{2 N(N+k) !(N-k) !}}, \\
\left|W_{m n}\right| & \leq \frac{1}{\sqrt{N}} e^{-k^{2} / 2 N} .
\end{aligned}
$$


Proof. We only need to obtain the asymptotics in $(2.13,2.14)$. This is an exercise in Stirling's formula:

$$
n !=\left(\frac{n}{e}\right)^{n} \sqrt{2 \pi n}\left(1+\frac{1}{12 n}+\frac{1}{288 n^{2}}+\ldots\right)
$$

or its log version

$$
\log n !=\left(n+\frac{1}{2}\right) \log n-n+\log \sqrt{2 \pi}+\ldots
$$

Here it is more convenient to use the latter. Using $(2.12,2.16)$,

$$
\begin{aligned}
& \log \frac{(m+n) !}{2^{m+n}\left(\frac{m+n}{2}\right) !}=\log \frac{(2 N) !}{N !}-\log 2^{2 N} \\
= & N \log N-N+\frac{1}{2} \log 2+\mathcal{O}\left(N^{-1}\right) .
\end{aligned}
$$

So

$$
\frac{(m+n) !}{2^{m+n}\left(\frac{m+n}{2}\right) !}=\left[1+\mathcal{O}\left(\frac{1}{N}\right)\right] \frac{N !}{\sqrt{\pi N}}
$$

using (2.17). Hence

$$
W_{m n}=\left[1+\mathcal{O}\left(\frac{1}{N}\right)\right] \frac{(-1)^{\frac{n-m}{2}} N !}{\sqrt{2 N(N+k) !(N-k) !}}, \quad N \gg 1
$$

which is (2.13). Using the fact that

$$
n !=\sqrt{2 \pi n}\left(\frac{n}{e}\right)^{n} e^{\lambda_{n}}
$$

with

$$
\frac{1}{12 n+1}<\lambda_{n}<\frac{1}{12 n}, \text { for all } n \geq 1,
$$

and applying the inequalities (with $x=k / N)$ :

$$
\phi(x) \stackrel{\text { def }}{=}(1+x) \log (1+x)+(1-x) \log (1-x) \geq x^{2}
$$

for all $x \in[0,1)$ and $\phi(x) \geq a x^{2}$ with $a>1$ for $x \in[7 / 10,1)$, we obtain (2.14). (When $x=k / N=1,(2.14)$ follows by a direct computation using Stirling's formula.)

From (2.14), the matrix element $W_{m n}$ has subexponential decay

$$
\left|W_{m n}\right| \leq \frac{1}{\sqrt{N}} e^{-\frac{|m-n|^{2}}{2 N}} \quad\left(N=\frac{m+n}{2} \gg 1\right)
$$

when $|m-n|>\sqrt{N}$. When $|m-n| \leq \sqrt{N}$, we only have the estimate

$$
\left|W_{m n}\right| \leq \frac{1}{\sqrt{N}}
$$

Hence $W$ is a matrix with a slowly enlarging region of size $\mathcal{O}(\sqrt{N})$ around the principal diagonal $W_{n n}$, the $\ell^{2}$ norm of $W$ is of $\mathcal{O}(1)$, but the local $\ell^{2}$ to $\ell^{\infty}$ norm is of order 
$\mathcal{O}(1 / \sqrt{N})$. These new features will need to be taken into account when we do the analysis in sections 3,4 .

\section{(2.1) in the Hermite-Fourier basis}

In the Hermite-Fourier basis, $e^{-i n \cdot \phi} h_{j}(x), n \in \mathbb{Z}^{\nu}, \phi \in \mathbb{T}^{\nu}, j \in\{0,1 \ldots\}$, the Floquet Hamiltonian, which is unitarily equivalent to the $K$ defined in (1.15) is then

$$
H=\operatorname{diag}\left(n \cdot \omega+j+\frac{1}{2}\right)+\frac{\delta}{2} W \otimes \Delta
$$

on $\ell^{2}\left(\mathbb{Z}^{\nu} \times\{0,1 \ldots\}\right)$, where $W$ is the matrix operator defined in (2.11), acting on the $j$ indices, $j=0,1,2 \ldots, \Delta$ acts on the $n$ indices, $n \in \mathbb{Z}^{\nu}$,

$$
\begin{aligned}
\Delta_{n n^{\prime}} & =1, & & \left|n-n^{\prime}\right|_{\ell^{1}}=1 \\
& =0, & & \text { otherwise. }
\end{aligned}
$$

Let $\tilde{H}=H-1 / 2$ and rename $\tilde{H}, H$; let $\tilde{\delta}=\delta / 2$ and rename $\tilde{\delta}, \delta$. We then have

$$
H \stackrel{\text { def }}{=} \operatorname{diag}(n \cdot \omega+j)+\delta W \otimes \Delta
$$

on $\ell^{2}\left(\mathbb{Z}^{\nu} \times\{0,1 \ldots\}\right)$, with $W, \Delta$ defined in $(2.11,2.22)$.

\section{Exponential decay of Green's functions at fixed $E$ : estimates in $\theta$}

Let $H$ be the operator defined in $(2.23)$, i.e.,

$$
H=\operatorname{diag}(n \cdot \omega+j)+\delta W \otimes \Delta
$$

on $\ell^{2}\left(\mathbb{Z}^{\nu} \times\{0,1, \ldots\}\right)$, where

$$
n \in \mathbb{Z}^{\nu}, j \in\{0,1, \ldots\}, 0<\delta \ll 1 .
$$

$W$ acts on the $j$ indices, $j=0,1,2 \ldots$,

$$
\begin{aligned}
W_{j j^{\prime}} & =\frac{(-1)^{\frac{j-j^{\prime}}{2}}}{2^{j+j^{\prime}} \sqrt{j ! j^{\prime} !}} \cdot \frac{\left(j+j^{\prime}\right) !}{\left(\frac{j+j^{\prime}}{2}\right) !}, & & j, j^{\prime} \text { same parity, } \\
& =0 & & \text { otherwise. }
\end{aligned}
$$

Write

$$
J=\frac{j+j^{\prime}}{2}, k=\frac{j-j^{\prime}}{2},\left(\text { assume } j \geq j^{\prime}\right) \text {. }
$$

When $J \gg 1$,

$$
\begin{aligned}
\left|W_{j j^{\prime}}\right| & \leq \frac{J !}{\sqrt{J(J+k) !(J-k) !}} \\
& \leq \frac{1}{\sqrt{J}} e^{-\frac{k^{2}}{2 J}}
\end{aligned}
$$


from (2.14). $\Delta$ acts on the $n$ indices, $n \in \mathbb{Z}^{\nu}$,

$$
\begin{aligned}
\Delta_{n n^{\prime}} & =1, & & \left|n-n^{\prime}\right|_{\ell^{1}}=1 \\
& =0, & & \text { otherwise. }
\end{aligned}
$$

In view of the Theorem, our aim is to prove that on a good set of $\omega, H$ has pure point spectrum. To achieve that goal, we add a parameter $\theta(\theta \in \mathbb{R})$ to $H$ :

$$
\begin{aligned}
H(\theta) & =H+\theta \\
& =\operatorname{diag}(n \cdot \omega+\theta+j)+\delta W \otimes \Delta .
\end{aligned}
$$

We consider a sequence of finite volume Green's functions at fixed $E$ :

$$
G_{\Lambda}(\theta, E)=\left(H_{\Lambda}(\theta)-E\right)^{-1}
$$

where $\Lambda$ are cubes in $\mathbb{Z}^{\nu} \times\{0,1 \ldots\}, \Lambda \nearrow \mathbb{Z}^{\nu} \times\{0,1 \ldots\}$ in an appropriate way, $H_{\Lambda}(\theta)$ are $H(\theta)$ restricted to $\Lambda$.

In this section, $E \in \mathbb{R}, \omega \in[0,2 \pi)^{\nu}$ are fixed and we assume that $\omega$ is a Diophantine frequency. We do estimates in $\theta$. Specifically, we prove (inductively) that for any $\Lambda$ large enough, away from a set of $\theta$ of small measure in $\mathbb{R},\left\|G_{\Lambda}(\theta, E)\right\|$ is bounded and $\left|G_{\Lambda}(\theta, E)(x, y)\right|$ has subexponential decay for $|x-y| \sim$ linear scale of $\Lambda$. (For precise statement, see Proposition 3.10.) As mentioned in section 1, the proof is a combination of a non perturbative part in the $n$ direction and a perturbatuve part in the $j$ direction. We note that this set of bad $\theta$ depends on $E, \omega$, the estimate on the measure is, howevere, uniform in $E$ and $\omega$ for Diophantine $\omega$.

In the next section (section 4 ), we eliminate the $E$ dependence by excluding double resonances and converting the estimate in $\theta$ into estimates in $\omega$ in the process. The conversion is possible because $\omega, \theta$ appear in (3.6) in the form $n \cdot \omega+\theta$.

Below we start the induction process. To simplify notations, we extend $H$ to a linear operator on $\ell^{2}\left(\mathbb{Z}^{\nu+1}\right)$ with $W_{j j^{\prime}}$ as in $(3.3)$ for $j, j^{\prime} \in\{0,1 \ldots\}$ and $W_{j j^{\prime}}=0$ otherwise.

\subsection{The initial estimate $\left(0^{\text {th }}\right.$ step).}

For any subset $\Lambda \subset \mathbb{Z}^{\nu+1}$, we define

$$
\begin{aligned}
H_{\Lambda}(\theta)\left(n, j ; n^{\prime}, j^{\prime}\right) & =H(\theta)\left(n, j ; n^{\prime}, j^{\prime}\right), & & \left(n, j ; n^{\prime}, j^{\prime}\right) \in \Lambda \times \Lambda, \\
& =0, & & \text { otherwise }
\end{aligned}
$$

Let $\Lambda_{0}=[-J, J]^{\nu+1}$ for some $J>0$ to be determined. For a fixed $E$, we study the Green's function $G_{\Lambda_{0}}(\theta, E)=\left(H_{\Lambda_{0}}(\theta)-E\right)^{-1}$ by doing perturbation theory in $\delta$. We have

Lemma 3.1. Assume $0<\delta \ll 1$. For any fixed $\sigma, 0<\sigma<1 / 4$, there exists $J \in \mathbb{N}$ such that the following statement is satisfied. Let $\Lambda_{0}=[-J, J]^{\nu+1}$. There exists a set $\mathcal{B}\left(\Lambda_{0}, E\right)$ in $\mathbb{R}$, with

$$
\text { mes } \mathcal{B}\left(\Lambda_{0}, E\right) \leq e^{-J^{\sigma / 2}} \text {, }
$$


such that if $\theta \in \mathbb{R} \backslash \mathcal{B}\left(\Lambda_{0}, E\right)$, then

$$
\begin{aligned}
& \left\|G_{\Lambda_{0}}(\theta, E)\right\| \leq e^{J^{\sigma}} \\
& \left|G_{\Lambda_{0}}(\theta, E)\left(n, j ; n^{\prime} j^{\prime}\right)\right| \leq e^{-\left|(n, j)-\left(n^{\prime}, j^{\prime}\right)\right|^{1 / 4}}, \quad\left|(n, j)-\left(n^{\prime}, j^{\prime}\right)\right|>J / 10 .
\end{aligned}
$$

Proof. Since this is the initial estimate, we do perturbation theory in $\delta$. Let $\kappa>0$. Let $\mathcal{B}\left(\Lambda_{0}, E\right)$ be the set such that if $\theta \in \mathcal{B}\left(\Lambda_{0}, E\right)$, then

$$
|n \cdot \omega+j+\theta-E| \leq 2 \kappa
$$

for some $(n, j) \in \Lambda_{0}$. Clearly

$$
\begin{aligned}
\operatorname{mes} \mathcal{B}\left(\Lambda_{0}, E\right) & \leq 4\left|\Lambda_{0}\right| \kappa \\
& =4(2 J+1)^{\nu+1} \kappa .
\end{aligned}
$$

Let

$$
D_{\Lambda_{0}}(\theta) \stackrel{\text { def }}{=} \operatorname{diag}(n \cdot \omega+j+\theta),(n, j) \in \Lambda_{0}
$$

be the unperturbed diagonal operator. Since $\|\delta W \otimes \Delta\|_{\ell^{2}} \leq \mathcal{O}(\delta)$, if $\theta \notin \mathcal{B}\left(\Lambda_{0}, E\right)$, then

$$
\begin{aligned}
& \left\|G_{\Lambda_{0}}(\theta, E)\right\|=\left\|\left(D_{\Lambda_{0}}(\theta)-E+\delta W \otimes \Delta\right)^{-1}\right\| \\
& \leq \kappa^{-1}
\end{aligned}
$$

for $\kappa \gg \delta$.

From the resolvent equation

$$
G_{\Lambda_{0}}(\theta, E)\left(n, j ; n^{\prime} j^{\prime}\right)=(n \cdot \omega+j+\theta-E)^{-1}\left[(\delta W \otimes \Delta) G_{\Lambda_{0}}(\theta, E)\right]\left(n, j ; n^{\prime} j^{\prime}\right)
$$

for $(n, j) \neq\left(n^{\prime}, j^{\prime}\right)$. Hence

$$
\left|G_{\Lambda_{0}}(\theta, E)\left(n, j ; n^{\prime} j^{\prime}\right)\right| \leq \mathcal{O}(1) \delta \kappa^{-2}, \text { if }\left|(n, j)-\left(n^{\prime}, j^{\prime}\right)\right|>J / 10
$$

Let

$$
\begin{aligned}
& J=|\log \delta|\left(\text { hence } \delta=e^{-J}\right), \\
& \kappa=e^{-J^{\sigma}} .
\end{aligned}
$$

(3.13-3.17) then imply (3.9-3.11).

\subsection{A Wegner estimate in $\theta$ for all scales.}

We now state an apriori estimate in $\theta$ for $\left\|\left(H_{\Lambda}(\theta)-E\right)^{-1}\right\|$ valid for all finite subsets $\Lambda \subset \mathbb{Z}^{\nu+1}$ and all $\delta$. This estimate will be useful in the induction process. Following the Anderson localization tradition, we call it a Wegner estimate. 
Proposition 3.2. For any $E \in \mathbb{R}$, and any finite subset $\Lambda$ in $\mathbb{Z}^{\nu+1}$, the following estimate is satisfied for all $\kappa>0$ :

$$
\text { mes }\left\{\theta \mid \operatorname{dist}\left(E, \sigma\left(H_{\Lambda}(\theta)\right)\right) \leq \kappa\right\} \leq 2|\Lambda| \kappa \text {. }
$$

Proof. Let $\lambda_{k}, k=1, \ldots,|\Lambda|$ be eigenvalues of $H_{\Lambda}(\theta=0)$. Then

$$
\left\{\theta \mid \operatorname{dist}\left(E, \sigma\left(H_{\Lambda}(\theta)\right)\right) \leq \kappa\right\}=\cup_{k=1}^{|\Lambda|}\left\{\theta|| E-\theta-\lambda_{k} \mid \leq \kappa\right\}
$$

It follows that the measure of the left side is bounded by $2|\Lambda| \kappa$.

Our goal now is to obtain inductively the equivalent of estimates (3.9-3.11) for larger subsets $\Lambda, \mathbb{Z}^{\nu+1} \supset \Lambda \supset \Lambda_{0}$. We note that in proving Lemma 3.1, we did perturbation theory about the diagonal operator

$$
D_{\Lambda_{0}}(\theta)=\left.\operatorname{diag}(n \cdot \omega+j+\theta)\right|_{(n, j) \in \Lambda_{0}}
$$

using the smallness of $\delta$. This was sufficient for one initial scale. For subsequent scales, however, we need more detailed information on the spectrum of $H_{\Lambda}$.

\subsection{Local spectral property of $H_{\Lambda}$.}

We assume that $\omega$ is Diophantine, i.e., $\exists c>0, \alpha>2 \nu$, such that

$$
|n \cdot \omega+j| \geq \frac{c}{|n|^{\alpha}}
$$

for all $n \in \mathbb{Z}^{\nu} \backslash\{0\}$, all $j \in \mathbb{Z}$. In this subsection, we make statements which hold for any fixed $\theta$. We look at finite subsets $\Lambda \subset \mathbb{Z}^{\nu+1}$, such that $j \neq 0$, if $(n, j) \in \Lambda$. When $j \gg 1$, this is the perturbative region.

Proposition 3.3. Assume $\omega$ satisfies (3.23). Let $0<\beta^{\prime}<1 / 5 \alpha$ and $3 / 4<\beta<1$. Let $\Lambda$ be a rectangle centered at $(N, 2 L) \in \mathbb{Z}^{\nu} \times \mathbb{Z}$, where $\mathbb{Z}^{\nu}$ is identified with $\mathbb{Z}^{\nu} \times\{0\}$ :

$$
\Lambda=(N, 2 L)+\left[-L^{\beta^{\prime}}, L^{\beta^{\prime}}\right]^{\nu} \times\left[-L^{\beta}, L^{\beta}\right] \subset \mathbb{Z}^{\nu+1}
$$

Assume

$$
|L| \gg 1
$$

For any fixed $\theta \in \mathbb{R}$, the eigenvalues $\lambda_{n, j}(\theta)$ of $H_{\Lambda}(\theta)$ satisfy

$$
\left|\lambda_{n, j}(\theta)-\lambda_{n^{\prime}, j^{\prime}}(\theta)\right|>\frac{1}{L^{1 / 5}} \quad(n, j) \neq\left(n^{\prime} j^{\prime}\right)
$$

the eigenfunctions $\phi_{n, j}$ may be chosen such that

$$
\left\|\phi_{n, j}-\delta_{n, j}\right\|<\frac{1}{L^{1 / 20}}
$$


Remark. It is crucial to note that the estimates $(3.26,3.27)$ are independent of $\theta$ and the specific $\omega$ satisfying (3.23). In Lemma 3.5, we exploit further the consequences of (3.26).

Proof of Proposition 3.3. Let $(n, j),\left(n^{\prime}, j^{\prime}\right) \in \Lambda,(n, j) \neq\left(n^{\prime}, j^{\prime}\right)$. Then for all $\theta$, the difference of the diagonal elements

$\left|n \cdot \omega+j+\theta-\left(n^{\prime} \cdot \omega+j^{\prime}+\theta\right)\right|=\left|\left(n-n^{\prime}\right) \cdot \omega+\left(j-j^{\prime}\right)\right| \geq \frac{c}{L^{\beta^{\prime} \alpha}}>\frac{1}{L^{1 / 5}}$ for $0<\beta^{\prime}<1 / 5 \alpha, L \gg 1$,

from (3.23). Use as approximate eigenfunctions $\delta_{n, j}$ with approximate eigenvalues $\tilde{\lambda}_{n, j}(\theta)=n \cdot \omega+j+\theta,(n, j) \in \Lambda$, and let

$$
\left(H_{\Lambda}-\tilde{\lambda}_{n, j}\right) \delta_{n, j}=\psi
$$

Then from (3.7),

$$
\begin{aligned}
\psi\left(n^{\prime}, j^{\prime}\right) & =\delta W_{j^{\prime} j}, & & \left|n-n^{\prime}\right|=1,\left(n^{\prime}, j^{\prime}\right) \in \Lambda \\
& =0, & & \text { otherwise },
\end{aligned}
$$

and

$$
\|\psi\|_{\ell^{2}}=\mathcal{O}\left(|L|^{-1 / 4}\right)
$$

from (3.4).

Equations (3.28-3.31) imply that $\tilde{\lambda}_{n, j}(\theta)=n \cdot \omega+j+\theta$ is an approximate eigenvalue of $H_{\Lambda}$ to $\mathcal{O}\left(|L|^{-1 / 4}\right)$. This can be seen as follows. Let $\lambda=\tilde{\lambda}_{n, j}(\theta)$. Assume

$$
\operatorname{dist}\left(\lambda, \sigma\left(H_{\Lambda}(\theta)\right)\right)>\mathcal{O}\left(|L|^{-1 / 4}\right) .
$$

Take any $f,\|f\|_{\ell^{2}(\Lambda)}=1, f=\sum c_{m} \psi_{m}, \sum\left|c_{m}\right|^{2}=1$, where $\psi_{m}$ are eigenfunctions of $H_{\Lambda}$. Then

$$
\left(H_{\Lambda}-\lambda\right) f=\sum\left(E_{m}-\lambda\right) c_{m} \psi_{m},
$$

where $E_{m}$ is the corresponding eigenvalue for $\psi_{m}$. Hence

$$
\left\|\left(H_{\Lambda}-\lambda\right) f\right\|=\sqrt{\sum\left(E_{m}-\lambda\right)^{2}\left|c_{m}\right|^{2}}>\mathcal{O}\left(|L|^{-1 / 4}\right) .
$$

This is a contradiction if $f=\delta_{n, j}$.

Hence (3.28) gives (3.26). (3.27) follows from $(3.26,3.31)$ and standard perturbation theory, see e.g., [Ka].

\subsection{The first iteration $\left(1^{\text {st }}\right.$ step $)$.}

We now increase the scale from $J$ to $J^{C}$, where $C>1$ (independent of $\delta$ ) is the geometric expansion factor, which will be specified in section 3.5. Recall that $J$ is the

initial scale, large enough so that (3.18) holds. Hence on $\Lambda_{0}=[-J, J]^{\nu+1},(3.9-3.11)$ hold. 
Let

$$
\Lambda \stackrel{\text { def }}{=}\left[-J^{C}, J^{C}\right]^{\nu+1} \text {. }
$$

Our aim is to prove the analogue of (3.9-3.11) when $\Lambda_{0}$ is replaced by $\Lambda$. The general strategy in going from scale $J$ to scale $J^{C}$ is to distinguish the region near $j=0$, where we use the estimate from the previous scale, here (3.9-3.11) and non perturbative arguments and the region away from $j=0$, where we use Proposition 3.3 and perturbation theory. The general iteration strategy here is similar to that in [BW1].

Toward that end, we define

$$
\mathcal{T} \stackrel{\text { def }}{=}\{(n, j) \in \Lambda|| j \mid \leq 2 J-1\}
$$

Let

$$
\Lambda_{0}(n, j)=(n, j)+[-J, J]^{\nu+1}, \quad(n, j) \in \mathcal{T}
$$

be cubes of the previous scale. Let

$\Lambda_{*}(n, 2 j)=(n, 2 j)+\left[-j^{\beta^{\prime}}, j^{\beta^{\prime}}\right]^{\nu} \times\left[-j^{\beta}, j^{\beta}\right] \quad\left(0<\beta^{\prime}<1 / 5 \alpha, 3 / 4<\beta<1\right), \quad(n, 2 j) \in \Lambda \backslash \mathcal{T}$,

be cubes of type (3.24). We cover $\Lambda$ with $\Lambda_{0}, \Lambda_{*}$ cubes, i.e., $\mathcal{T}$ with $\Lambda_{0}, \Lambda \backslash \mathcal{T}$ with $\Lambda_{*}$. $G_{\Lambda}$ are then obtained by using resolvent equation, (3.9-3.11), Propositions 3.2 and 3.3. We implement this strategy in our first iteration. This iteration is special, as in the tube region $\mathcal{T}$, we use smallness of $\delta$, cf. (3.18).

We need the following notion of pairwise disjointness. Let $S_{k}, k=1, \ldots, K$ be finite sets , $S_{k} \neq S_{k^{\prime}}$ if $k \neq k^{\prime}$. Let $S=\left\{S_{k}\right\}_{k=1}^{K}$. If $S_{k} \cap S_{k^{\prime}} \neq \emptyset, \forall k \neq k^{\prime}$, then we say there is 1 pairwise disjoint set in $S$. More generally, if $\exists I_{1}, I_{2}, \ldots, I_{P}, I_{p} \cap I_{p^{\prime}}=\emptyset$, if $p \neq p^{\prime},\left\{I_{p}\right\}_{p=1}^{P}=\{1,2, \ldots, K\}$ such that $S_{k} \cap S_{k^{\prime}} \neq \emptyset$ if and only if $k, k^{\prime} \in I_{p}$ for some $p$. Then we say there are $P$ pairwise disjoint sets in $S$.

Lemma 3.4. Let $\Gamma_{0}$ be a covering of $\mathcal{T}$ with $\Lambda_{0}$ cubes defined in (3.35). Assume $\omega$ is Diophantine satisfying (3.23). Fix $E, \sigma(0<\sigma<1 / 4)$ as in Lemma 3.1. For all $\theta$, there exists at most 1 pairwise disjoint $\tilde{\Lambda} \in \Gamma_{0}$, such that

$$
\operatorname{dist}\left(E, \sigma\left(H_{\tilde{\Lambda}}(\theta)\right)\right) \leq e^{-J^{\sigma}} \text {. }
$$

Moreover if $\Lambda_{0} \in \Gamma_{0}$ and $\Lambda_{0} \cap \tilde{\Lambda}=\emptyset$,

$$
\operatorname{dist}\left(E, \sigma\left(H_{\Lambda_{0}}(\theta)\right)\right) \geq \frac{c}{J^{C \alpha}} .
$$

Proof. Let $\Lambda_{0} \in \Gamma_{0}$. If $\left|\lambda_{n}-E\right|<e^{-J^{\sigma}}$ for a $\lambda_{n} \in \sigma\left(H_{\Lambda_{0}}(\theta)\right.$, then since $\|\delta W \otimes \Delta\| \leq$ $C \delta=C e^{-J}$,

$$
|n \cdot \omega+j+\theta-E| \leq e^{-J^{\sigma}}+C e^{-J} \leq 2 e^{-J^{\sigma}}
$$

for some $(n, j) \in \Lambda_{0}$. Thus if both $\Lambda_{0}$ and $\tilde{\Lambda}$ satisfy (3.37), then

$$
\left|\left(n-n^{\prime}\right) \cdot \omega+j-j^{\prime}\right| \leq|n \cdot \omega+j+\theta-E|+\left|n^{\prime} \cdot \omega+j^{\prime}+\theta-E\right| \leq 4 e^{-J^{\sigma}}
$$


for some $(n, j) \in \Lambda_{0}$ and $\left(n^{\prime}, j^{\prime}\right) \in \tilde{\Lambda}$. This implies $\Lambda_{0} \cap \tilde{\Lambda} \neq \emptyset$ for large $J$, since otherwise the left side is larger than $c /\left(2 \nu J^{C}\right)^{\alpha}$ by the Diophantine condition (3.23), which is a contradiction if $C \ll J^{\sigma} / \log J$.

If $\Lambda_{0} \in \Gamma_{0}$ is disjoint from $\tilde{\Lambda} \in \Gamma_{0}$, then for any $(n, j) \in \Lambda_{0}$ and for some $\left(n^{\prime}, j^{\prime}\right) \in \tilde{\Lambda}$,

$$
|n \cdot \omega+j+\theta-E| \geq\left|\left(n-n^{\prime}\right) \cdot \omega+j-j^{\prime}\right|-\left|n^{\prime} \cdot \omega+j^{\prime}+\theta-E\right| \geq \frac{c}{\left(2 \nu J^{C}\right)^{\alpha}}-2 e^{-J^{\sigma}} .
$$

Since $\|\delta W \otimes \Delta\| \leq C \delta=C e^{-J},(3.38)$ follows.

If $\Lambda_{0} \cap \tilde{\Lambda}=\emptyset$, then $(3.10,3.11)$ are available. Let $\Gamma_{*}$ be a covering of $\Lambda \backslash \mathcal{T}$. For $\Lambda_{*} \in \Gamma_{*}$, we need

Lemma 3.5. Let $0<\beta^{\prime}<1 / 5 \alpha$ and $3 / 4<\beta<1$. Let $\Lambda_{*}$ be the rectangle:

$$
\Lambda_{*}(n, 2 j)=(n, 2 j)+\left[-j^{\beta^{\prime}}, j^{\beta^{\prime}}\right]^{\nu} \times\left[-j^{\beta}, j^{\beta}\right], \quad(n, 2 j) \in \Lambda \backslash \mathcal{T} .
$$

For a fixed $E \in \mathbb{R}$, there exists $W \subset \mathbb{R}$, with mes $W \leq C\left|\Lambda_{*}\right| e^{-J^{\beta^{\prime} / 2}}$ such that for $\theta \in \mathbb{R} \backslash W, J \gg 1$,

$$
\begin{aligned}
& \left|\left[H_{\Lambda_{*}}(\theta)-E\right]^{-1}\left(n^{\prime}, j^{\prime} ; n^{\prime \prime}, j^{\prime \prime}\right)\right| \leq e^{-\frac{1}{2}\left[\left|n^{\prime}-n^{\prime \prime}\right|+\frac{\mid j^{\prime}-j^{\prime \prime}}{j^{1 / 2}}\right]}, \\
& \left(\left|n^{\prime}-n^{\prime \prime}\right|>j^{\beta^{\prime}} / 10, \text { or }\left|j^{\prime}-j^{\prime \prime}\right| \geq j^{\beta} / 10\right) \\
& \left\|\left[H_{\Lambda_{*}}(\theta)-E\right]^{-1}\right\| \leq e^{J^{\beta^{\prime} / 2}} .
\end{aligned}
$$

Proof. For any fixed $\theta, E$, there exists at most 1 bad site $b=\left(n^{\prime}, j^{\prime}\right) \in \Lambda_{*}$, such that

$$
\left|n^{\prime} \cdot \omega+j^{\prime}+\theta-E\right| \leq \frac{1}{j^{1 / 5}} .
$$

This is because if there were $\left(n^{\prime}, j^{\prime}\right),\left(n^{\prime \prime}, j^{\prime \prime}\right) \in \Lambda_{*},\left(n^{\prime}, j^{\prime}\right) \neq\left(n^{\prime \prime}, j^{\prime \prime}\right)$ such that

$$
\begin{aligned}
& \left|n^{\prime} \cdot \omega+j^{\prime}+\theta-E\right| \leq \frac{1}{j^{1 / 5}}, \\
& \left|n^{\prime \prime} \cdot \omega+j^{\prime \prime}+\theta-E\right| \leq \frac{1}{j^{1 / 5}},
\end{aligned}
$$

then

$$
\left|\left(n^{\prime}-n^{\prime \prime}\right) \cdot \omega+\left(j^{\prime}-j^{\prime \prime}\right)\right| \leq \frac{2}{j^{1 / 5}},
$$

which contradicts the Diophantine condition (3.23) on $\omega$ :

$$
\left\|\left(n^{\prime}-n^{\prime \prime}\right) \cdot \omega\right\|_{\mathbb{T}} \geq \frac{c}{\left|n^{\prime}-n^{\prime \prime}\right|^{\alpha}} \geq \frac{c}{j^{\beta^{\prime} \alpha}} \gg \frac{2}{j^{1 / 5}}
$$

for $j \geq J \gg 1$, using (3.41). 
To obtain $(3.42,3.43)$, we first consider $H_{\Lambda_{*} \backslash b}(\theta)$ and make estimates on $\left[H_{\Lambda_{*} \backslash b}(\theta)-\right.$ $E]^{-1}\left(n^{\prime}, j^{\prime} ; n^{\prime \prime}, j^{\prime \prime}\right)$. We then use Proposition 3.2 and the resolvent equation to obtain (3.42). We prove below that

$$
\left\|\left[H_{\Lambda_{*} \backslash b}(\theta)-E\right]^{-1}\right\|_{\ell^{2} \rightarrow \ell^{2}}<2 j^{1 / 5},
$$

for $j \gg 1$. To obtain estimates on the matrix elements, we use weighted $\ell^{2}$ space and show that $\left[H_{\Lambda_{*} \backslash b}(\theta)-E\right]^{-1}$ remain bounded. (3.45) is then the special case when the weight equals to 1 .

Toward that end, for any $p \in \mathbb{Z}^{\nu+1}$, we define $|p|_{\nu}=\left|\left(p_{1}, \ldots, p_{\nu}, 0\right)\right|$ and $|p|_{1}=$ $\left|\left(0, \ldots, 0, p_{\nu+1}\right)\right|$. Let $\rho=\left\{\rho_{a}\right\}_{a \in \mathbb{Z}^{\nu+1}}$ be a family of weights, such that

$$
\rho_{a}(p)=e^{|p-a|_{\nu}+\frac{|p-a|_{1}}{j^{1 / 2}}} \quad\left(a \in \mathbb{Z}^{\nu+1}\right), \quad \forall p \in \mathbb{Z}^{\nu+1},
$$

where $j$ is as in (3.41). Hence $\forall p, q \in \mathbb{Z}^{\nu+1}$,

$$
\begin{aligned}
\rho_{a}^{-1}(p) \rho_{a}(q) & =e^{-|p-a|_{\nu}-\frac{|p-a|_{1}}{j^{1 / 2}}} \cdot e^{|q-a|_{\nu}+\frac{|q-a|_{1}}{j^{1 / 2}}} \\
& \leq e^{|p-q|_{\nu}+\frac{|p-q|_{1}}{j^{1 / 2}}}
\end{aligned}
$$

for all $\rho_{a} \in \rho$.

Let $D$ be the diagonal part in (3.8). To arrive at (3.42), we consider the deformed operator $\tilde{H}_{\Lambda_{*} \backslash b}(\theta)$ :

$$
\begin{aligned}
\tilde{H}_{\Lambda_{*} \backslash b}(\theta)(p, q) \stackrel{\text { def }}{=}\left[\rho_{a}^{-1} H_{\Lambda_{*} \backslash b}(\theta) \rho_{a}\right](p, q) \\
=D_{p q}+\delta \rho_{a}^{-1}(p) \rho_{a}(q)(W \otimes \Delta)_{p q} \\
=D_{p q}+\delta(\tilde{W} \otimes \tilde{\Delta})_{p q}
\end{aligned}
$$

where $p, q \in \Lambda_{*} \backslash b$ and

$$
(\tilde{W} \otimes \tilde{\Delta})_{p q} \stackrel{\text { def }}{=} \rho_{a}^{-1}(p) \rho_{a}(q)(W \otimes \Delta)_{p q}
$$

To prove boundedness of $\left[\tilde{H}_{\Lambda_{*} \backslash b}(\theta)-E\right]^{-1}$, we use resolvent series and perturb about the diagonal. We have formally

$$
\begin{aligned}
{\left[\tilde{H}_{\Lambda_{*} \backslash b}(\theta)-E\right]^{-1} } & =(D-E)^{-1}+\delta(D-E)^{-1}(\tilde{W} \otimes \tilde{\Delta})(D-E)^{-1} \\
& +\delta^{2}(D-E)^{-1}(\tilde{W} \otimes \tilde{\Delta})(D-E)^{-1}(\tilde{W} \otimes \tilde{\Delta})(D-E)^{-1} \\
& +\ldots
\end{aligned}
$$

Let

$$
\mathcal{W}^{\text {def }}=(\tilde{W} \otimes \tilde{\Delta})(D-E)^{-1}
$$

Then

$$
\begin{aligned}
\mathcal{W}\left(n^{\prime}, j^{\prime} ; n^{\prime \prime}, j^{\prime \prime}\right) & =\tilde{W}_{j^{\prime} j^{\prime \prime}} \tilde{\Delta}_{n^{\prime} n^{\prime \prime}}\left(n^{\prime \prime} \cdot \omega+j^{\prime \prime}+\theta-E\right)^{-1}, \quad\left|n^{\prime}-n^{\prime \prime}\right|=1 \\
& =0 \quad \text { otherwise. }
\end{aligned}
$$


We bound $\mathcal{W}$ using Schur's lemma $[\mathrm{Ka}]$ :

$$
\|\mathcal{W}\| \leq\left(\sup _{n^{\prime}, j^{\prime}} \sum_{n^{\prime \prime}, j^{\prime \prime}}\left|\mathcal{W}\left(n^{\prime}, j^{\prime} ; n^{\prime \prime}, j^{\prime \prime}\right)\right|\right)^{1 / 2}\left(\sup _{n^{\prime \prime}, j^{\prime \prime}} \sum_{n^{\prime}, j^{\prime}}\left|\mathcal{W}\left(n^{\prime}, j^{\prime} ; n^{\prime \prime}, j^{\prime \prime}\right)\right|\right)^{1 / 2},
$$

where $\left(n^{\prime}, j^{\prime}\right),\left(n^{\prime \prime}, j^{\prime \prime}\right) \in \Lambda_{*} \backslash b$.

$$
\sum_{n^{\prime \prime}, j^{\prime \prime}}\left|\mathcal{W}\left(n^{\prime}, j^{\prime} ; n^{\prime \prime}, j^{\prime \prime}\right)\right|=\sum_{\left|n^{\prime \prime}-n^{\prime}\right|=1} \sum_{\left|j^{\prime \prime}-2 j\right| \leq j^{\beta}}\left|\tilde{W}_{j^{\prime} j^{\prime \prime}} \tilde{\Delta}_{n^{\prime} n^{\prime \prime}}\left(n^{\prime \prime} \cdot \omega+j^{\prime \prime}+\theta-E\right)^{-1}\right|,
$$

$\left(n^{\prime}, j^{\prime}\right) \in \Lambda_{*} \backslash b$. From $(3.47,3.4)$

$$
\begin{aligned}
\left|\tilde{W}_{j^{\prime} j^{\prime \prime}} \tilde{\Delta}_{n^{\prime} n^{\prime \prime}}\right| & \leq \frac{e}{\sqrt{j}} e^{\left[-\frac{\left(j^{\prime}-j^{\prime \prime}\right)^{2}}{2\left(j^{\prime}+j^{\prime \prime}\right)}+\frac{\left|j^{\prime}-j^{\prime \prime}\right|}{j^{1 / 2}}\right]} \\
& \leq \frac{e^{200}}{\sqrt{j}} e^{-\frac{\left(j^{\prime}-j^{\prime \prime}\right)^{2}}{10 j}}
\end{aligned}
$$

where we used $j^{\prime}+j^{\prime \prime} \leq 4 j+2 j^{\beta} \leq 9 j / 2$ for $j^{\prime}, j^{\prime \prime} \in \Lambda_{*} \backslash b$. So

$$
\begin{aligned}
\sum_{n^{\prime \prime}, j^{\prime \prime}}\left|\mathcal{W}\left(n^{\prime}, j^{\prime} ; n^{\prime \prime}, j^{\prime \prime}\right)\right| & \leq \frac{e^{200}}{\sqrt{j}} \sum_{\left|n^{\prime \prime}-n^{\prime}\right|=1} \sum_{\left|j^{\prime \prime}-2 j\right| \leq j^{\beta}}\left|n^{\prime \prime} \cdot \omega+j^{\prime \prime}+\theta-E\right|^{-1} \\
& \leq \frac{\mathcal{O}(1)}{\sqrt{j}}\left(j^{1 / 5}+\log j\right) .
\end{aligned}
$$

Let $a_{j^{\prime \prime}} \stackrel{\text { def }}{=} n^{\prime \prime} \cdot \omega+j^{\prime \prime}+\theta-E$. To arrive at (3.54), we used the fact that $\left|a_{j^{\prime \prime}}\right|=$ $\left|n^{\prime \prime} \cdot \omega+j^{\prime \prime}+\theta-E\right|>j^{-1 / 5}$ for all $\left(n^{\prime \prime}, j^{\prime \prime}\right) \in \Lambda_{*} \backslash b$ and that $a_{p}-a_{q}=p-q$, for all $p, q$.

Using (3.53), we have

$$
\sum_{n^{\prime}, j^{\prime}}\left|\mathcal{W}\left(n^{\prime}, j^{\prime} ; n^{\prime \prime}, j^{\prime \prime}\right)\right| \leq \mathcal{O}(1) j^{1 / 5}
$$

Substituting $(3.54,3.55)$ into $(3.52)$, we have

$$
\begin{aligned}
\|\mathcal{W}\| & \leq \mathcal{O}(1)\left(\frac{j^{2 / 5}}{j^{1 / 2}}\right)^{1 / 2} \\
& <\mathcal{O}(1) j^{-1 / 20} \quad(j \gg 1) .
\end{aligned}
$$

So the Neumann series in (3.50) is norm convergent:

$$
\begin{aligned}
\left\|\left[\tilde{H}_{\Lambda_{*} \backslash b}(\theta)-E\right]^{-1}\right\| & \leq\left\|(D-E)^{-1}\right\|\left(1+\delta\|\mathcal{W}\|+\delta^{2}\|\mathcal{W}\|^{2}+\ldots\right) \\
& <2\left\|(D-E)^{-1}\right\| \\
& <2 j^{1 / 5}, \quad 22
\end{aligned}
$$


which is equivalent to

$$
\left\|\rho_{a}\left(H_{\Lambda_{*} \backslash b}(\theta)-E\right)^{-1} \rho_{a}^{-1}\right\|<2 j^{1 / 5} .
$$

For each pair $p, q \in \Lambda_{*} \backslash b$, we can then always choose $a$ so that

$$
\left|\left[H_{\Lambda_{*} \backslash b}(\theta)-E\right]^{-1}\left(n^{\prime}, j^{\prime} ; n^{\prime \prime}, j^{\prime \prime}\right)\right| \leq C j^{1 / 5} e^{-\left[\left|n^{\prime}-n^{\prime \prime}\right|+\frac{\left|j^{\prime}-j^{\prime \prime}\right|}{j^{1 / 2}}\right]} .
$$

Write $G_{0}$ for $\left[H_{\Lambda_{*} \backslash b}(\theta)-E\right]^{-1}, G$ for $\left[H_{\Lambda_{*}}(\theta)-E\right]^{-1}$. From the resolvent equation:

$$
G=G_{0}+G_{0} H_{b} G_{0}+G_{0} H_{b} G H_{b} G_{0}
$$

where $H_{b}=H_{\Lambda_{*}}-H_{\Lambda_{*} \backslash b}$. The matrix element $H_{b}(p, q)=0$, unless $p=b$ or $q=b$. Using this, (3.57), the Wegner estimate (3.20) on $G$ with $\kappa=e^{-J^{\beta^{\prime} / 2}}\left(0<\beta^{\prime}<1 / 5 \alpha\right)$ and self-adjointness, we obtain $(3.42,3.43)$

We now write the estimate at scale $J^{C}$. Assume $\delta, J$ satisfying (3.18), so that Lemma 3.1 holds. Let $J_{1}=J^{C}, C>1$, the same geometric expansion factor as before. Let $\Lambda=\left[-J_{1}, J_{1}\right]^{\nu+1}$. We have

Lemma 3.6. Assume $\omega$ is Diophantine satisfying (3.23) and $0<\delta \ll 1$ is the same as in Lemma 3.1. For any fixed $\sigma, 0<\sigma<1 / 5 \alpha$, there exists $\mathcal{B}(\Lambda, E)$ in $\mathbb{R}$, with

$$
\text { mes } \mathcal{B}(\Lambda, E) \leq e^{-J_{1}^{\sigma / 2}}
$$

such that if $\theta \in \mathbb{R} \backslash \mathcal{B}(\Lambda, E)$, then

$$
\begin{aligned}
& \left\|G_{\Lambda}(\theta, E)\right\| \leq e^{J_{1}^{\sigma}} \\
& \left|G_{\Lambda}(\theta, E)\left(n, j ; n^{\prime} j^{\prime}\right)\right| \leq e^{-\left|(n, j)-\left(n^{\prime}, j^{\prime}\right)\right|^{1 / 4}}, \\
& \quad \text { for all }(n, j),\left(n^{\prime} j^{\prime}\right) \text { such that }\left|(n, j)-\left(n^{\prime}, j^{\prime}\right)\right|>J_{1} / 10,
\end{aligned}
$$

provided the expansion factor $C$ satisfies $1<C<\beta^{\prime} / \sigma, 0<\beta^{\prime}<1 / 5 \alpha$ is as in (3.41).

So we have the same estimate as in Lemma 3.1 at the larger scale $J_{1}=J^{C}(C>1)$.

Proof. This is similar to the proof of Lemma 2.4 in [BW1]. So we summarize the main steps. We prove $(3.59,3.60)$ using the resolvent equation and cover $\Lambda$ with cubes of types $\Lambda_{0}, \Lambda_{*}$ defined in $(3.35,3.36)$, i.e., $\mathcal{T}$, defined in $(3.34)$, with a covering $\Gamma_{0}$ of $\Lambda_{0}$ 's and $\Lambda \backslash \mathcal{T}$, a covering $\Gamma_{*}$ of $\Lambda_{*}$ 's. From Lemma 3.4, for all fixed $E$, all $\theta$, there exists at most 1 pairwise disjoint $\Lambda_{0} \in \Gamma_{0}$ on which $(3.10,3.11)$ do not hold. We use Proposition 3.2 on this $\Lambda_{0},(3.10,3.11)$ on all other $\Lambda_{0}$.

For a given $\Lambda_{*}$, let $W_{\Lambda_{*}}$ be the set such that $(3.42,3.43)$ hold if $\theta \in \mathbb{R} \backslash W_{\Lambda_{*}}$. Let $\mathcal{W}=\cup W_{\Lambda_{*}}$, where the union is over all possible $\Lambda_{*}$ with centers in $\Lambda \backslash \mathcal{T}$.

$$
\operatorname{mes} \mathcal{W} \leq \mathcal{O}(1) J_{1}^{2(\nu+1)} e^{-J^{\beta^{\prime} / 2}} \leq e^{-J_{1}^{\frac{\beta^{\prime}}{2 C}}} \cdot J_{1}^{2(\nu+1)}
$$


where the first term is an upper bound on the number of possible $\Lambda_{*}$ with centers in $\Lambda \backslash \mathcal{T}$ multiplied by the volume of $\Lambda_{*}$. For $\theta \in \mathbb{R} \backslash \mathcal{W}$, we use $(3.42,3.43)$ on $\Lambda_{*}$.

Using the resolvent equation, combining (3.10, 3.11, 3.42, 3.43) and (3.20) with $\kappa=e^{-J_{1}^{\sigma}}(0<\sigma<1 / 5 \alpha)$ on the only bad $\Lambda_{0}$, we obtain $(3.59,3.60)$. (For more general iterations using the resolvent equation, cf. proof of Lemma 3.8, in particular (3.72).) Combining the measure estimate from (3.20) with $\kappa=e^{-J_{1}^{\sigma}}$ and the above measure estimate on $\mathcal{W}$, we obtain (3.58), provided $C<\beta^{\prime} / \sigma$.

For the induction process to follow, it is convenient to define the following. For any fixed $\sigma, 0<\sigma<1 / 5 \alpha$ and any given box $\Lambda \subset \mathbb{Z}^{\nu+1}$ of side length $2 J+1$, we say $G_{\Lambda}(\theta, E)$ at fixed $(\theta, E)$ is good if

$$
\begin{aligned}
& \left\|G_{\Lambda}(\theta, E)\right\| \leq e^{J^{\sigma}} \\
& \quad\left|G_{\Lambda}(\theta, E)\left(n, j ; n^{\prime} j^{\prime}\right)\right| \leq e^{-\left|(n, j)-\left(n^{\prime}, j^{\prime}\right)\right|^{1 / 4}}, \\
& \quad \forall(n, j),\left(n^{\prime} j^{\prime}\right) \text { such that }\left|(n, j)-\left(n^{\prime}, j^{\prime}\right)\right|>J / 10 .
\end{aligned}
$$

Otherwise, it is bad. We also define

$$
\mathcal{W}(\Lambda) \stackrel{\text { def }}{=} \bigcup W_{\Lambda_{*}}
$$

where the union is over all possible $\Lambda_{*}$ of the form (3.36) with centers in $\Lambda \backslash \mathcal{T}$ and $\mathcal{T}$ is as defined in (3.34), so that for $\theta \in \mathbb{R} \backslash \mathcal{W}(\Lambda),(3.42,3.43)$ are valid for all $\Lambda_{*}$ with centers in $\Lambda \backslash \mathcal{T}$. We say $\Lambda_{*}$ is good, if $(3.42,3.43)$ hold. Otherwise $\Lambda_{*}$ is bad.

\subsection{A large deviation estimate in $\theta$ for the Green's functions at fixed $E$ at all scales.}

We now increase the scale from $J_{1}$ to $J_{1}^{C}\left(1<C<\beta^{\prime} / \sigma\right.$, the geometric expansion factor will be determined here). Our task is again to derive estimates (3.58-3.60) for the cube $\left[-J_{1}^{C}, J_{1}^{C}\right]^{\nu+1}$ starting from the estimates $(3.58-3.60)$ for the cube $\left[-J_{1}, J_{1}\right]^{\nu+1}$.

For simplicity of notation, we rename $J_{1}, J$ and $\left[-J_{1}^{C}, J_{1}^{C}\right]^{\nu+1}, \Lambda$ in this section. As in the first iteration, we distinguish the tube region $\mathcal{T}$, defined as in (3.34) with the new $J$. We cover $\mathcal{T}$ with $\Lambda_{0}$ 's defined in (3.35) with the new $J$, and $\Lambda \backslash \mathcal{T}, \Lambda_{*}$ defined in (3.36). We note that $\Lambda_{0}$ are at scale $J$ with centers in $\mathcal{T}$, while $\Lambda_{*}$ are at scales from $J^{\beta^{\prime}}$ to $J^{C \beta}\left(0<\beta^{\prime}<1 / 5 \alpha, 3 / 4<\beta<1\right)$ with centers away from $\mathcal{T}$.

As in the first iteration, we use the resolvent equation to obtain estimates on $G_{\Lambda}$ from estimates on $G_{\Lambda_{0}}$ and $G_{\Lambda_{*}}$. Let $\Gamma_{0}$ be a covering of $\mathcal{T}$ and $\Gamma_{*}$ of $\Lambda \backslash \mathcal{T}$. For $\theta \in \mathbb{R} \backslash \mathcal{W}(\Lambda),(3.42,3.43)$ are valid on all $\Lambda_{*} \in \Gamma_{*}$. So for any fixed $\theta \in \mathbb{R} \backslash \mathcal{W}(\Lambda)$, we only need to control the number of pairwise disjoint bad $\Lambda_{0}$ boxes on which estimate (3.61) is not available. In particular, we need the number of such bad boxes to be $\ll J^{C}$, the linear scale of the box $\Lambda$. (This is intuitively clear, as otherwise without further detail on the location of the bad boxes, we could not accumulate decay at the linear scale as in (3.60).) Recall that for the first iteration, there is at most 1 such (pairwise disjoint) bad box. 
Lemma 3.7. Assume $\omega$ is Diophantine, satisfying (3.23) and Lemma 3.6 is valid on cubes

$$
\Lambda_{0}(0, j)=(0, j)+[-J, J]^{\nu+1}, \forall j \in[-(2 J-1), 2 J-1] .
$$

Then for all fixed $\theta$

$$
\begin{aligned}
& \#\left\{(n, j) \in \mathcal{T} \mid \Lambda_{0}(n, j) \text { is a bad box }\right\} \\
& \leq \mathcal{O}(1) J^{5(\nu+1)}=\left(J^{C}\right)^{1-} \ll J^{C},
\end{aligned}
$$

by choosing $5(\nu+1)<C \ll J^{\sigma / 2}(0<\sigma<1 / 5 \alpha)$.

Proof. Write $\Lambda_{0}$ for $\Lambda_{0}(0, j)$. We first replace the estimate $\left\|G_{\Lambda_{0}}(\theta, E)\right\|_{\ell^{2} \rightarrow \ell^{2}} \leq e^{J_{1}^{\sigma}}$ in (3.59) by the estimate on the Hilbert-Schmidt norm:

$$
\left\|G_{\Lambda_{0}}(\theta, E)\right\|_{\mathrm{HS}} \leq e^{J_{1}^{\sigma}}
$$

This leaves the measure estimate in (3.58) unchanged for $J_{1} \gg 1$ (cf. proof of Lemma $3.6)$.

Define

$$
\mathcal{A} \stackrel{\text { def }}{=} \bigcup_{j \in[-(2 J-1), 2 J-1]} \mathcal{B}\left(\Lambda_{0}(0, j)\right) .
$$

Since the conditions on the Green's function in $\left(3.59^{\prime}, 3.60\right)$ can be rewritten as polynomial inequalities in $\theta$ by using Cramer's rule, $\mathcal{A}$ is semi-algebraic of total degree less than

$$
\begin{aligned}
& (2 J+1)^{2(\nu+1)} \cdot(2 J+1)^{2(\nu+1)} \cdot(4 J+1) \\
& \leq \mathcal{O}_{\nu}(1) J^{5(\nu+1)},
\end{aligned}
$$

where the first factor is an upper bound of the degree of polynomial for each entry of the matrix $G_{\Lambda_{0}}(\theta, E)$, the second is an upperbound on the \# of entries of each $G_{\Lambda_{0}}(\theta, E)$ plus the one for the Hilbert-Schmidt norm, the third is the \# of different matrices $G_{\Lambda_{0}}$ 's. For more details, cf. the proof of Lemma 2.6 in [BW1]. $\mathcal{A}$ is therefore the union of at most $\mathcal{O}_{\nu}(1) J^{5(\nu+1)}$ intervals in $\mathbb{R}$ by using Theorem 1 in [Ba] (see also [BGS], where the special case we need is restated as Theorem 7.3).

For any fixed $\theta \in \mathbb{R}$, let

$$
I=\left\{n \in\left[-J^{C}, J^{C}\right]^{\nu} \mid n \cdot \omega+\theta \in \mathcal{A}\right\} .
$$

Then $\Lambda_{0}(n, j)$ is a bad box if and only if $n \in I$ and, therefore, $n$ is in one of the intervals of $\mathcal{A}$. But each interval does not contain two such $n$ if $\omega$ satisfies (3.23) and $|I| \leq \mathcal{O}_{\nu}(1) J^{5(\nu+1)}$ by virtue of (3.58). This is because for Diophantine $\omega$ satisfying (3.23), if there exist $n, n^{\prime} \in\left[-J^{C}, J^{C}\right]^{\nu}, n \neq n^{\prime}$, then

$$
\left|\left(n-n^{\prime}\right) \cdot \omega\right| \geq \frac{c}{\left(2 J^{C}\right)^{\alpha}} \gg e^{-J^{\sigma / 2}} .
$$

Hence each interval can contain at most 1 integer point in $\left[-J^{C}, J^{C}\right]^{\nu}$. We therefore obtain (3.64).

For any fixed $\theta \in \mathbb{R} \backslash \mathcal{W}(\Lambda), \mathcal{W}(\Lambda)$ defined as in (3.62), the only bad boxes are of type $\Lambda_{0}$. Lemma 3.7 shows that there are only few (of order $\left(\left(J^{C}\right)^{1-}\right)$ bad $\Lambda_{0}$ boxes in $\Lambda$. The following iteration lemma will enable us to obtain estimates (3.58-3.60) for $G_{\Lambda}$ at scale $J^{C}$. 
Lemma 3.8. Fix $b \in(0,1 / 8)$ and assume $\tau$ satisfies $3 / 4+b<\tau<1-b$. Suppose $M, N$ are integers satisfying

$$
N^{\tau} \leq M \leq 2 N^{\tau}
$$

Let $\Lambda=[-N, N]^{\nu+1}$. Assume for all $\bar{\Lambda} \subseteq \Lambda$ with diameter $L$, the Green's function $G_{\bar{\Lambda}}(E)=\left(H_{\bar{\Lambda}}-E\right)^{-1}$ at energy $E$ satisfies

$$
\left\|G_{\bar{\Lambda}}\right\| \leq e^{L^{b}}
$$

Let $\Lambda^{\prime}$ be cubes of side length $2 M$. We say that $\Lambda^{\prime}$ is good if in addition to (3.69'), the Green's function exhibits off-diagonal decay:

$$
\left|G_{\Lambda^{\prime}}(E)(x, y)\right| \leq e^{-|x-y|^{1 / 4}}
$$

for all $x, y \in \Lambda^{\prime}$ satisfying $|x-y|>M / 10$. Otherwise $\Lambda^{\prime}$ is bad. Assume for any family $\mathcal{F}$ of pair-wise disjoint bad $\Lambda^{\prime}$ cubes in $\Lambda$,

$$
\# \mathcal{F} \leq N^{b}
$$

Under these assumptions, one has

$$
\left|G_{\Lambda}(E)(x, y)\right| \leq e^{-|x-y|^{1 / 4}}
$$

for all $x, y \in \Lambda$ satisfying $|x-y| \geq N / 10$, provided $N$ is sufficiently large, i.e., $N \geq$ $N_{0}(b, \tau)$.

Proof. The proof is similar to the proof of Lemma 2.4 in [BGS]. As we will see from $(3.73,3.74)$, because of the conditions on $b, \tau$, it only needs a one step iteration.

To estimate $G_{\Lambda}(E)(x, y), x, y \in \Lambda,|x-y| \geq N / 10$, let $Q$ be cubes of side length $4 M$, we make an exhaustion $\left\{S_{i}(x)\right\}_{i=0}^{\ell}$ of $\Lambda$ of width $2 M$ centered at $x$ as follows:

$$
\begin{aligned}
& S_{-1}(x) \stackrel{\text { def }}{=} \emptyset, \\
& S_{0}(x) \stackrel{\text { def }}{=} \Lambda^{\prime}(x) \cap \Lambda, \\
& S_{i}(x) \stackrel{\text { def }}{=} \cup_{y \in S_{i-1}} Q(y) \cap \Lambda,
\end{aligned}
$$

for $1 \leq i \leq \ell$, where $\ell$ is maximal such that $S_{\ell}(x) \neq \Lambda$.

We say an annulus $A_{i}=S_{i}(x) \backslash S_{i-1}(x)$ is good if $A_{i} \cap \mathcal{F}=\emptyset$. Let $A_{i}(x), A_{i+1}(x), \ldots, A_{i+s}(x)$ be adjacent good annuli and define

$$
U=\cup_{k=i}^{i+s} A_{k}(x)
$$

Let $\partial_{*} S_{-1}(x)=\{x\}$ and

$$
\partial_{*} S_{j}(x)=\left\{y \in S_{j}(x)\left|\exists z \in \Lambda \backslash S_{j}(x),\right| y-z \mid=1\right\}
$$

for $j \geq 0$. 
By construction

$$
\operatorname{dist}\left(\partial_{*} S_{i-1}, \partial_{*} S_{i+s}\right)=2 M(s+1) \quad(s \geq 0) .
$$

For any subset $B \subseteq \Lambda$, let $H_{B}$ be defined as in (3.8). Let

$$
\Gamma_{B} \stackrel{\text { def }}{=} H_{\Lambda}-\left(H_{B} \oplus H_{\Lambda \backslash B}\right)
$$

Assume $x \in B$, from the resolvent equation,

$$
\begin{aligned}
G_{\Lambda}(E)(x, y) & =G_{B}(E)(x, y) \\
+ & \sum_{\substack{z \in B \\
z^{\prime} \in \Lambda \backslash B}} G_{B}(E)(x, z) \Gamma_{B}\left(z, z^{\prime}\right) G_{\Lambda}(E)\left(z^{\prime}, y\right) .
\end{aligned}
$$

The proof follows the same line of arguments as in the proof of Lemma 2.4 in [BGS] by iterating (3.70). There are two modifications:

- In view of $\Gamma_{B}$, with matrix elements $\Gamma_{B}\left(z, z^{\prime}\right)=\delta W\left(j, j^{\prime}\right) \Delta\left(n, n^{\prime}\right), z=(n, j) \in B$, $z^{\prime}=\left(n^{\prime}, j^{\prime}\right) \in \Lambda \backslash B$, where $\Delta\left(n, n^{\prime}\right)$ as defined in (2.22) and

$$
\left|W\left(j, j^{\prime}\right)\right| \leq e^{-\frac{\left(j-j^{\prime}\right)^{2}}{2\left(j+j^{\prime}\right)}} \leq e^{-\frac{\left(j-j^{\prime}\right)^{2}}{4 N}}
$$

from (2.14) for $1 \ll j+j^{\prime} \leq 2 N$, we define

$$
\begin{aligned}
& \partial S_{i}^{(1)}(x)=\left\{y \in S_{i}(x) \mid \operatorname{dist}\left(y, \partial_{*} S_{i}(x)\right) \leq N^{11 / 16}\right\} \\
& \partial S_{i}^{(2)}(x)=\left\{y \in \Lambda \backslash S_{i}(x) \mid \operatorname{dist}\left(y, \partial_{*} S_{i}(x)\right) \leq N^{11 / 16}\right\}
\end{aligned}
$$

We note that for $y \in \partial S_{i}^{(1)}(x) \cup \partial S_{i}^{(2)}(x)$, $\operatorname{dist}\left(y, \partial_{*} S_{i}(x)\right) \leq N^{11 / 16} \ll M$, the size of $\Lambda^{\prime}$ cubes, and for $j, j^{\prime}$ such that $\left|j-j^{\prime}\right|>N^{11 / 16}$,

$$
\left|W\left(j, j^{\prime}\right)\right| \ll e^{-N^{1 / 4}}
$$

- For all $x \in U$, with $\operatorname{dist}\left(x, \partial_{*} S_{i-1}\right) \geq M / 4$, there exists $x^{\prime} \in U$ such that $\Lambda^{\prime}\left(x^{\prime}\right) \subset$ $U$ and $\operatorname{dist}\left(x, \partial_{*} \Lambda^{\prime}\left(x^{\prime}\right)\right) \geq M / 5$. We estimate $G_{U}(E)(x, y)$ with $x \in \partial S_{i-1}^{(2)}, y \in$ $\partial S_{i+s}^{(1)} \operatorname{using} \Lambda^{\prime}\left(x^{\prime}\right) \subset U$.

To obtain subexponential decay of off-diagonal elements $G_{\Lambda}(E)(x, y)$ we proceed as in [BGS]. This entails to estimate iteratively $G_{S_{n_{i}}}(E)(x, z)$, where $z \in \partial S_{n_{i}}^{(1)}$ and $A_{n_{i}}=S_{n_{i}} \backslash S_{n_{i}-1}$ is a good annulus. Let $n_{i}<m_{i}<n_{i+1}$, so that all the annulus in $S_{m_{i}} \backslash S_{n_{i}}$ are bad and all the annulus in $S_{n_{i+1}} \backslash S_{m_{i}}=U$ are good. 
Using the resolvent equation to relate $G_{S_{n_{i+1}}}(E)(x, z), z \in \partial S_{n_{i+1}}^{(1)}$ with $G_{S_{n_{i}}}(E)(x, y)$, $y \in \partial S_{n_{i}}^{(1)}$, we have

$$
\begin{aligned}
G_{S_{n_{i+1}}}(E)(x, z)=\sum_{\substack{w \in U \\
w^{\prime} \in S_{n_{i+1}} \backslash U}} G_{U}(E)(z, w) \Gamma_{U}\left(w, w^{\prime}\right) G_{S_{n_{i+1}}}(E)\left(w^{\prime}, x\right) \\
=\sum_{\substack{w \in \partial S_{m_{i}}^{(2)} \\
w^{\prime} \in \partial S_{m_{i}}^{(1)}}} G_{U}(E)(z, w) \Gamma_{U}\left(w, w^{\prime}\right) G_{S_{n_{i+1}}}(E)\left(w^{\prime}, x\right)+o\left(e^{-N^{1 / 4}}\right) \\
=\sum_{\substack{w \in \partial S_{m_{i}}^{(2)} w^{\prime} \in \partial S_{m_{i}}^{(1)} \\
y \in \partial S_{n_{i}}^{(1)} y^{\prime} \in \partial S_{n_{i}}^{(2)}}} G_{U}(E)(z, w) \Gamma_{U}\left(w, w^{\prime}\right) G_{S_{n_{i}}}(E)(y, x) \Gamma_{S_{n_{i}}}\left(y, y^{\prime}\right) G_{S_{n_{i+1}}}(E)\left(y^{\prime}, w^{\prime}\right) \\
+o\left(e^{-N^{1 / 4}}\right),
\end{aligned}
$$

where we used $(3.71,2.22)$. This is the analogue of $(2.25,2.26)$ in [BGS]. Iterating (3.72) and taking the log log, lead to the conditions

$$
\begin{gathered}
\log \log \left[\left(e^{N^{b}}\right)^{N / M}\right]<\log \log e^{N^{1 / 4}}, \\
\log \log e^{\left[M^{1 / 4}\left(N / M-N^{b}\right)\right]}>\log \log e^{N^{1 / 4}},
\end{gathered}
$$

for $N \gg 1$, where (3.73) originates from the estimates on $\left\|G_{S_{n_{i+1}}}\right\|$ and (3.74) from the decay estimates on $G_{U} \cdot(3.73,3.74)$ in turn lead to $3 / 4+b<\tau<1-b$ for $M, N$ satisfying (3.69) (cf.[BGS]).

In order to apply the above lemma, we need to convert the covering of $\Lambda=$ $\left[-J^{C}, J^{C}\right]^{\nu+1}$ which is of diverging scales from $J^{\beta^{\prime}}\left(0<\beta^{\prime}<1 / 5 \alpha\right)$ to $J^{C \beta}(3 / 4<$ $\beta<1)$ to a covering of a single scale $J^{C \beta}$. Let $\Lambda^{\prime}$ be cubes at scale $J^{C \beta}(3 / 4<\beta<1)$, i.e.,

$$
\Lambda^{\prime}(n, j)=(n, j)+\left[-J^{C \beta}, J^{C \beta}\right]^{\nu+1}, \quad(n, j) \in \mathbb{Z}^{\nu+1} .
$$

Let $\mathcal{W}$ be as in (3.62). We define

$\Lambda^{\prime}$ bad (for a fixed $\theta \in \mathbb{R} \backslash \mathcal{W}(\Lambda)$ ), if $\Lambda^{\prime} \cap \tilde{\Lambda} \neq \emptyset$, where $\tilde{\Lambda}$ is a bad $\Lambda_{0}$ box, i.e., (3.61) is violated.

Let $\Gamma^{\prime}$ be a covering of $\Lambda$ with $\Lambda^{\prime}$ boxes defined in (3.75). Lemma 3.7 gives

Lemma 3.9. Fix $\sigma \in(0,1 / 5 \alpha)$ as in Lemma 3.6. For any fixed $\theta \in \mathbb{R} \backslash \mathcal{W}(\Lambda)$, $\Gamma^{\prime}$ has at most $\mathcal{O}(1) J^{5(\nu+1)}=\left(J^{C}\right)^{1-} \ll J^{C}\left(5(\nu+1)<C \ll J^{\sigma / 2}\right)$ pairwise disjoint bad $\Lambda^{\prime}$ boxes. On the good $\Lambda^{\prime}$ box, we have

$$
\begin{aligned}
& \left\|G_{\Lambda^{\prime}}(\theta)\right\| \leq e^{\left(J^{C \beta}\right)^{\sigma}} \\
& \left|G_{\Lambda^{\prime}}\left(\theta ; n, j ; n^{\prime} j^{\prime}\right)\right| \leq e^{-\left|(n, j)-\left(n^{\prime}, j^{\prime}\right)\right|^{1 / 4}} \\
& \quad \forall(n, j),\left(n^{\prime} j^{\prime}\right) \text { such that }\left|(n, j)-\left(n^{\prime}, j^{\prime}\right)\right|>J^{C \beta} / 10
\end{aligned}
$$


provided

$$
\max \left(5(\nu+1), \frac{\beta^{\prime}}{2 \beta \sigma}\right)<C<\frac{\beta^{\prime}}{\sigma} \ll J^{\sigma / 2} .
$$

So (3.61) is satisfied for the good $\Lambda^{\prime}$.

Proof. From (3.64), there are at most $\mathcal{O}(1) J^{5(\nu+1)}$ points $(n, j) \in \mathcal{T}$, such that $\Lambda_{0}(n, j)$ is a bad box, where the $\mathcal{O}(1)$ only depends on the dimension $\nu$. Since the $\Lambda^{\prime}$ boxes are at scale $J^{C \beta} \gg J$, the scale of $\Lambda_{0}$ boxes, there are at most $2^{\nu+1}$ non intersecting $\Lambda^{\prime}$ boxes intersecting a given bad $\Lambda_{0}$ box. Hence for any fixed $\theta \in \mathbb{R} \backslash \mathcal{W}(\Lambda), \Gamma^{\prime}$ has at most $\mathcal{O}(1) J^{5(\nu+1)}$ pairwise disjoint bad $\Lambda^{\prime}$ boxes with a slightly larger $\mathcal{O}(1)$.

On the good $\Lambda^{\prime}$ boxes, we use the resolvent equation to obtain (3.77) as follows. We first estimate $\left\|G_{\Lambda^{\prime}}(\theta)\right\|$. Let $\epsilon>0$ be arbitrary. We have

$$
\begin{aligned}
G_{\Lambda}(E+i \epsilon)(x, y) & =G_{U(x)}(E+i \epsilon)(x, y) \\
+ & \sum_{\substack{z \in U(x) \\
z^{\prime} \in \Lambda \backslash U(x)}} G_{U(x)}(E+i \epsilon)(x, z) \Gamma_{U(x)}\left(z, z^{\prime}\right) G_{\Lambda}(E+i \epsilon)\left(z^{\prime}, y\right),
\end{aligned}
$$

where $\Gamma_{U(x)}$ is as defined above (3.70), $U(x)$ is either $\Lambda_{*}$ or $\Lambda_{0}$ and $x \in U(x)$. Write $U$ for $U(x)$. For $y^{\prime} \in \partial_{*} U=\left\{y^{\prime \prime} \in U|\exists z \in \Lambda \backslash U| z-,y^{\prime \prime} \mid=1\right\}, x$ and $y^{\prime}$ satisfy the distance condition in (3.42) if $U=\Lambda_{*}$ and the condition in (3.11) if $U=\Lambda_{0}$. It is easy to see that for all $x \in \Lambda$, such a $U(x)$ exists.

Summing over $y$, we have

$$
\begin{aligned}
\sum_{y}\left|G_{\Lambda}(E+i \epsilon)(x, y)\right| \leq \sum_{y \in U(x)}\left\|G_{U}\right\| \\
+2^{\nu+1} J^{C(\nu+1)} e^{-\frac{J^{\beta^{\prime}}}{11}} \delta \sup _{w} \sum_{y}\left|G_{\Lambda}(E+i \epsilon)(w, y)\right|,
\end{aligned}
$$

where we used $(2.14,2.22,3.10,3.11,3.42,3.43)$ and the conditions $\sigma, \beta^{\prime}<1 / 5 \alpha$, $\alpha>2 \nu$. Taking the supremum over $x$ and $U(x)$, we obtain the bound on $\left\|G_{\Lambda^{\prime}}(\theta)\right\|$ in (3.77), provided $C>\beta^{\prime} / 2 \beta \sigma, J \gg 1$.

Applying the resolvent equation one more time, similar to the proof of Lemma 3.8, and using the bound on $\left\|G_{\Lambda^{\prime}}(\theta)\right\|$, we obtain the off-diagonal decay in (3.77).

Let $0<\beta^{\prime}<1 / 5 \alpha,(\alpha$ as in $(3.23)), 3 / 4<\beta<1$. Choose $\sigma \in(0,1 / 5 \alpha)$ satisfying $5(\nu+1)<\beta^{\prime} / \sigma$, which leads to

$$
0<\sigma<\frac{\beta^{\prime}}{5(\nu+1)}<\frac{1}{25 \alpha(\nu+1)}
$$

Choosing appropriate $C$ large enough so that both Lemme 3.8 and 3.9 are available, we then arrive at the following estimate for $G_{\Lambda}(\theta)=\left(H_{\Lambda}(\theta)-E\right)^{-1}$ valid for all $\Lambda=[-J, J]^{\nu+1}$, with $J$ large enough and any fixed $E$. 
Proposition 3.10. Assume $\omega$ is Diophantine, satisfying (3.23). For any $0<\beta^{\prime}<$ $1 / 5 \alpha$, fix

$$
0<\sigma<\frac{\beta^{\prime}}{20(\nu+1)} .
$$

Then for all $0<\delta \ll 1$, there exists $J_{0}$ such that the following statement is satisfied for all $\Lambda=[-J, J]^{\nu+1}$, with $J \geq J_{0}$ : There exists $\mathcal{B}(\Lambda, E)$ in $\mathbb{R}$, with

$$
\text { mes } \mathcal{B}(\Lambda, E) \leq e^{-J^{\sigma / 2}},
$$

such that if $\theta \in \mathbb{R} \backslash \mathcal{B}(\Lambda, E)$, then

$$
\begin{aligned}
& \left\|G_{\Lambda}(\theta, E)\right\| \leq e^{J^{\sigma}} \\
& \left|G_{\Lambda}(\theta, E)\left(n, j ; n^{\prime} j^{\prime}\right)\right| \leq e^{-\left|(n, j)-\left(n^{\prime}, j^{\prime}\right)\right|^{1 / 4}}, \\
& \quad \text { for all }(n, j),\left(n^{\prime} j^{\prime}\right) \text { such that }\left|(n, j)-\left(n^{\prime}, j^{\prime}\right)\right|>J / 10 .
\end{aligned}
$$

Proof. Choose $C>40(\nu+1)$ such that

$$
\frac{\beta^{\prime}}{2 \beta}<C \sigma<\beta^{\prime}<1 \text {. }
$$

Assume $0<\delta \ll 1$. Let $J_{0}=|\log \delta|^{\frac{1}{C}}$. For the scales $J_{0}$ to $J_{0}^{C}$, we use perturbation theory in $\delta$ as in Lemma 3.1, with a further lowering of $\delta$ if necessary in order that (3.9-3.11) hold for all scales in $\left[J_{0}, J_{0}^{C}\right]$. For the scales $\geq J_{0}^{C}$, Lemme 3.8 and 3.9 are available, in view of the choice of $C$.

Assume (3.78-3.80) hold at some scale $J \gg 1$. Using Lemma 3.9 in Lemma 3.8, we obtain the corresponding estimate at scale $J^{C}$. Hence the proposition holds by induction.

\section{Frequency estimates and the elimination of $E$.}

We now convert the estimate in $\theta$ in (3.78-3.80) for fixed $E, \omega$ into estimates in $\omega$ for fixed $\theta(\theta=0)$, eliminating the $E$ dependence in the process by excluding double resonances. This is the key estimate leading to the proof of the Theorem in section 5 . We will first define a double resonant set in $(\omega, \theta) \in \mathbb{T}^{\nu} \times \mathbb{R}$.

We use 2 scales $N, \bar{N}$ with $N<\bar{N}, \log \log \bar{N} \ll \log N$. Let $\Lambda_{N}(j)=[-N, N]^{\nu+1}+$ $(0, j), j \in \mathbb{Z}, \Lambda_{\bar{N}}=[-\bar{N}, \bar{N}]^{\nu+1}$. Assume $\Lambda_{\bar{N}}$ is resonant at $\theta=0$ for some $E$, i.e., $E$ is close to some eigenvalue of $H_{\Lambda_{\bar{N}}}$ (see (4.1) below). We prove in Lemma 4.1 that with further reductions in the frequency set, the $\Lambda_{N}(j)$ boxes with $|j| \leq \bar{N}$ and are at appropriate distances from $\Lambda_{\bar{N}}$ are all nonresonant (at $\theta=0$ ).

Toward that end, let $D C(\bar{N})$ be the Diophantine condition to order $\bar{N}$, i.e., if $\omega \in D C(\bar{N})$, then

$$
\|n \cdot \omega\|_{\mathbb{T}} \geq \frac{c}{|n|^{\alpha}} \quad(\alpha>2 \nu), \forall n \in[-\bar{N}, \bar{N}]^{\nu} \backslash\{0\},
$$


where as before $\|\cdot\|_{\mathbb{T}}$ is the distance to the nearest integer. Denote $H_{\Lambda_{\bar{N}}}$ by $H_{\bar{N}}$. We define $\mathcal{D}(N, \bar{N}) \subset \mathbb{T}^{\nu} \times \mathbb{R}$ as

$$
\begin{array}{r}
\mathcal{D}(N, \bar{N})=\underset{\left\{j \in \mathbb{Z} \mid \Lambda_{N}(j) \cap \Lambda_{\bar{N}} \neq \emptyset\right\}}{\bigcup}\left\{(\omega, \theta) \in \mathbb{T}^{\nu} \times \mathbb{R} \mid \exists E,\right. \text { such that } \\
\left.\left\|\left(H_{\bar{N}}(\omega, 0)-E\right)^{-1}\right\| \geq e^{N^{2}} \text { and } \theta \in \mathcal{B}\left(\Lambda_{N}(j), E\right)\right\},
\end{array}
$$

and for fixed $0<a<1$ and $C>1$,

$$
S(N)=\bigcup_{\bar{N} \asymp N^{C}}\{\mathcal{D}(N, \bar{N}) \bigcap(D C(\bar{N}) \times \mathbb{R})\},
$$

where $\bar{N} \asymp N^{C}$ means $a N^{C} \leq \bar{N} \leq N^{C} / a$. We note that $a$ is kept fixed in this section.

The set $S(N)$ is the double resonant set in $(\omega, \theta)$ restricted to $\omega \in D C(\bar{N}), S(N) \subset$ $\mathbb{T}^{\nu} \times \mathbb{R}$. It is the projection of the set $\tilde{S}(N), \tilde{S}(N) \subset \mathbb{T}^{\nu} \times \mathbb{R} \times \mathbb{R}$, i.e., $S(N)=$ $\operatorname{Proj} \mathbb{T}^{\nu} \times \mathbb{R}(\tilde{S}(N))$ with

$$
\tilde{S}(N)=\bigcup_{\bar{N} \asymp N^{C}}\{\tilde{\mathcal{D}}(N, \bar{N}) \bigcap(D C(\bar{N}) \times \mathbb{R} \times \mathbb{R})\}
$$

where

$$
\begin{aligned}
\tilde{\mathcal{D}}(N, \bar{N})=\left\{(\omega, \theta, E) \in \mathbb{T}^{\nu} \times \mathbb{R} \times \mathbb{R} \mid\left\|\left(H_{\bar{N}}(\omega, 0)-E\right)^{-1}\right\| \geq e^{N^{2}}\right. \\
\left.\quad \text { and } \theta \in \mathcal{B}\left(\Lambda_{N}(j), E\right) \text { for some } \Lambda_{N}(j) \cap \Lambda_{\bar{N}} \neq \emptyset\right\}
\end{aligned}
$$

Lemma 4.1. Let $N \in \mathbb{N}$ be sufficiently large, $C>1$ and $\delta>0$ sufficiently small. Let $\Omega_{N}$ be the union of $\Omega_{\bar{N}}^{*}$ over $\bar{N} \asymp N^{C}$, where $\Omega_{\bar{N}}^{*} \subset D C(\bar{N})$ is the set of $\omega$, such that

- there exists $E \in \mathbb{R}$ such that

$$
\left\|\left(H_{\bar{N}}(\omega, 0)-E\right)^{-1}\right\| \geq e^{N^{2}}
$$

- there exist $j \in \mathbb{Z}, \Lambda_{N}(j) \cap \Lambda_{\bar{N}} \neq \emptyset$, and $\ell \in \mathbb{Z}^{\nu},|\ell| \asymp \exp \left[(\log N)^{2}\right]=K$ such that

$$
\left|\left(H_{\Lambda_{N}(j)}-E\right)^{-1}(\omega, \ell \cdot \omega)\left(m, m^{\prime}\right)\right|>e^{-\left|m-m^{\prime}\right|^{1 / 4}}
$$

for some $m, m^{\prime} \in \Lambda_{N}(j),\left|m-m^{\prime}\right|>N / 10$.

Then the set $\Omega_{N}$ satisfies

$$
\text { mes } \Omega_{N} \leq \exp \left[-\frac{1}{100}(\log N)^{2}\right]
$$

The proof of Lemma 4.1 uses the following decomposition lemma [B3, Lemma 9.9]. 
Lemma 4.2. Let $\mathcal{S} \subset[0,1]^{2 \nu}$ be a semi-algebraic set of degree $B$ and $\operatorname{mes}_{2 \nu} \mathcal{S}<$ $\eta, \log B \ll \log 1 / \eta$. Denote by $(x, y) \in[0,1]^{\nu} \times[0,1]^{\nu}$ the product variable. Fix $\epsilon>$ $\eta^{1 / 2 \nu}$. Then there is a decomposition

$$
\mathcal{S}=\mathcal{S}_{1} \bigcup \mathcal{S}_{2}
$$

with $\mathcal{S}_{1}$ satisfying

$$
\left|\operatorname{Proj}_{x} \mathcal{S}_{1}\right|<B^{C} \epsilon
$$

and $\mathcal{S}_{2}$ satisfying the transversality property

$$
\operatorname{mes}_{\nu}\left(\mathcal{S}_{2} \cap L\right)<B^{C} \epsilon^{-1} \eta^{1 / 2 \nu},
$$

for any $\nu$-dimensional hyperplane $L$ such that

$$
\max _{1 \leq j \leq \nu}\left|\operatorname{Proj}_{L}\left(e_{j}\right)\right|<\frac{1}{100} \epsilon
$$

where $e_{j}$ are the basis vectors for the $x$-coordinates.

For our usage, the variable $(x, y) \in \mathbb{R}^{2 \nu}$ in the lemma will be $(\omega, \theta)$ after identifying $\theta$ with $(\theta, 0) \in \mathbb{R}^{\nu}$.

Proof of Lemma 4.1. Let $(\omega, \theta) \in S(N)$, then $\theta \in \mathcal{B}\left(\Lambda_{N}(j), E_{k}\right)$ for some $\Lambda_{N}(j)$ and some eigenvalue $E_{k}$ of $H_{\bar{N}}$ satisfying $\left|E_{k}-E\right|<e^{-N^{2}}$. This is because the perturbation $e^{-N^{2}}$ essentially preserves the condition of the definition of a bad set, see (3.8-3.10) (c.f., proof of Lemma 4.1 [BW1]). By the Diophantine restrictions on the frequencies

$$
\text { mes } S(N) \leq \mathcal{O}(1) e^{-N^{\sigma / 2}} N^{C(\nu+2)}
$$

from Proposition 3.10, where the third factor in the RHS is an upper bound on the number of $\Lambda_{N}(j)$ and the number of eigenvalues of $H_{\bar{N}}$.

Since $\tilde{S}(N)$ is semi-algebraic with total degree at most $N^{C_{1} \nu}\left(C_{1}>1\right)$, cf., proof of Lemma 3.7, $S(N)$ its projection onto $\mathbb{T}^{\nu} \times \mathbb{R}$ is also semi-algebraic with degree at most $N^{C_{2} \nu}\left(C_{2}>C_{1}\right)$ [Chap 9, B3]. (4.8) and Lemma 4.2 then conclude the proof by taking $\epsilon \asymp \exp \left[-(\log N)^{2}\right]$ (cf., also [BW2]).

Here we also used the fact that the sum of the measure estimate in (4.9) over all $L$ corrsponding to the hyperplanes $(\omega, \ell \cdot \omega)$ with $|\ell| \asymp \exp \left[(\log N)^{2}\right]$ is much smaller than $\exp \left[-(\log N)^{2}\right]$ for the corresponding $B, \eta$ and $\epsilon>0$.

Lemma 4.1 shows that the boxes $\Lambda_{N}(\ell, j)=(\ell, j)+[-N, N]^{\nu+1}$, where $|\ell| \asymp$ $\exp \left[(\log N)^{2}\right], j \in\left[-N^{C}, N^{C}\right]$ are non resonant with the box $\Lambda_{\bar{N}}=[-\bar{N}, \bar{N}]^{\nu+1}$, $\bar{N} \asymp N^{C}$. Let $\mathcal{T}$ be the tube region,

$$
\mathcal{T}=\left\{(n, j) \in \mathbb{Z}^{\nu+1} \mid j \in\left[-N^{C}, N^{C}\right]\right\}
$$

The boxes $\Lambda_{N}(\ell, j)$ have centers in $\mathcal{T}$.

We now exclude resonances of $\Lambda_{\bar{N}}$ with boxes with centers in $\mathbb{Z}^{d} \backslash \mathcal{T}$. Recall from section 3 , that boxes with centers in $\mathbb{Z}^{d} \backslash \mathcal{T}$ are in fact rectangles:

$\Lambda_{*}(n, 2 j)=(n, 2 j)+\left[-j^{\beta^{\prime}}, j^{\beta^{\prime}}\right]^{\nu} \times\left[-j^{\beta}, j^{\beta}\right] \quad\left(0<\beta^{\prime}<1 / 5 \alpha, 3 / 4<\beta<1\right), \quad(n, 2 j) \in \mathbb{Z}^{d} \backslash \mathcal{T}$.

To exclude resonances of boxes $\Lambda_{*}(n, 2 j)$ where $\max (|n|,|2 j|) \asymp \exp \left[(\log N)^{2}\right]$ with $\Lambda_{\bar{N}}$, we use direct perturbation. We have 


\section{Lemma 4.3.}

mes $\left\{\omega \in[0,2 \pi)^{\nu} \mid \operatorname{dist}\left(\sigma\left(H_{\bar{N}}\right), \sigma\left(H_{\Lambda_{*}}\right)\right) \leq \kappa, \omega\right.$ satisfies (3.23) $\}$

$$
\leq C \kappa\left|\Lambda_{\bar{N}}\right|\left|\Lambda_{*}\right|, \quad\left(\kappa \ll \exp \left[-\frac{1}{5}(\log N)^{2}\right]\right)
$$

for any $\Lambda_{*}=\Lambda_{*}(n, 2 j)$ in (4.13) satisfying $|n|>\exp \left[\frac{1}{2}(\log N)^{2}\right]$ and $\max (|n|,|2 j|) \asymp$ $\exp \left[(\log N)^{2}\right]$, provided $0<\beta^{\prime}<\min (1 / 20,1 / 5 \alpha)$.

Proof. Let $\lambda_{m, k}, \phi_{m, k}$ be eigenvalues and eigenfunctions of $H_{\Lambda_{*}}$ :

$$
H_{\Lambda_{*}} \phi_{m, k}=\lambda_{m, k} \phi_{m, k}
$$

Write $\phi_{m, k}=\sum_{\left(m^{\prime}, k^{\prime}\right) \in \Lambda_{*}} a_{m^{\prime}, k^{\prime}} \delta_{m^{\prime}, k^{\prime}}$, with $\sum\left|a_{m^{\prime}, k^{\prime}}\right|^{2}=1$. Then

$$
\lambda_{m, k}=\sum_{\left(m^{\prime}, k^{\prime}\right)}\left|a_{m^{\prime}, k^{\prime}}\right|^{2}\left(m^{\prime} \cdot \omega+k^{\prime}\right)+\delta \sum_{\left(m^{\prime}, k^{\prime}\right),\left(m^{\prime \prime}, k^{\prime \prime}\right)} a_{m^{\prime}, k^{\prime}} \bar{a}_{m^{\prime \prime}, k^{\prime \prime}} \Delta_{m^{\prime} m^{\prime \prime}} W_{k^{\prime} k^{\prime \prime}} .
$$

From (3.27) of Proposition 3.3

$$
\sum_{\left(m^{\prime}, k^{\prime}\right) \neq(m, k)}\left|a_{m^{\prime}, k^{\prime}}\right|^{2}=\mathcal{O}\left(\frac{1}{j^{1 / 20}}\right) .
$$

The first order eigenvalue variation:

$$
\begin{aligned}
m \cdot \frac{\partial}{\partial \omega} \lambda_{m, k} & =\sum_{\left(m^{\prime}, k^{\prime}\right)}\left|a_{m^{\prime}, k^{\prime}}\right|^{2} m \cdot m^{\prime} \\
& =\sum_{\left(m^{\prime}, k^{\prime}\right)}\left|a_{m^{\prime}, k^{\prime}}\right|^{2} m \cdot m+\sum_{\left(m^{\prime}, k^{\prime}\right), m^{\prime} \neq m}\left|a_{m^{\prime}, k^{\prime}}\right|^{2} m \cdot\left(m^{\prime}-m\right) \\
& \geq|m|^{2}-\frac{\mathcal{O}(1)}{j^{1 / 20}}|m| \cdot j^{\beta^{\prime}}
\end{aligned}
$$

where we used (4.15) and the fact that $\left|m-m^{\prime}\right| \leq \mathcal{O}(1) j^{\beta^{\prime}}$ in $\Lambda_{*}$. Lowering $\beta^{\prime}$ to satisfy $\beta^{\prime}<1 / 20$ if necessary, we obtain

$$
m \cdot \frac{\partial}{\partial \omega} \lambda_{m, k} \geq \frac{1}{2}|m|^{2} \gg m N^{C}
$$

for $|m|>\exp \left[\frac{1}{2}(\log N)^{2}\right] \gg N^{C}$.

Let $\mu_{\ell, i}(\omega)$ be the eigenvalues of $H_{\bar{N}}$. Then $\mu_{\ell, i}(\omega)$ are piecewise holomorphic in each $\omega_{p}, p=1, \ldots, \nu$ and Lipshitz in $\omega:\left\|\mu_{\ell, i}\right\|_{\text {Lip }} \leq C \bar{N} \asymp N^{C}$. Using this and (4.16), we have that

$$
\begin{aligned}
& \quad \operatorname{mes}\left\{\omega \in[0,2 \pi)^{\nu}\left|\min _{\ell, i} \min _{m, k}\right| \lambda_{m, k}(\omega)-\mu_{\ell, i}(\omega) \mid \leq \kappa\right\} \\
& \leq C \kappa\left|\Lambda_{\bar{N}}\right|\left|\Lambda_{*}\right| .
\end{aligned}
$$

Using Lemma 4.3, we arrive at 
Lemma 4.4. Let $N \in \mathbb{N}$ be sufficiently large, $C>2 / \beta^{\prime}, 0<\beta^{\prime}<\min (1 / 20,1 / 5 \alpha)$ as in Lemma 4.3, $\delta$ sufficiently small. Let $\Omega_{N}^{\prime}$ be a subset of the Diophantine set defined in (3.23) with the properties:

- there are $\bar{N} \asymp N^{C}$ and $E$ such that

$$
\left\|\left(H_{\bar{N}}(\omega, 0)-E\right)^{-1}\right\| \geq e^{N^{2}} ;
$$

- there is a

$$
\tilde{\Lambda}(n, 2 j)=(n, 2 j)+\left[-j^{\beta}, j^{\beta}\right]^{\nu+1}, \quad(3 / 4<\beta<1)
$$

with $|j| \geq N^{C} / 2$ and $\max (|n|,|2 j|) \asymp \exp \left[(\log N)^{2}\right]$ such that

$$
\left|\left(H_{\tilde{\Lambda}(n, 2 j)}-E\right)^{-1}(\omega, 0)\left(m, m^{\prime}\right)\right|>e^{-\left|m-m^{\prime}\right|^{1 / 4}}
$$

for some $m, m^{\prime} \in \tilde{\Lambda}$ satisfying $\left|m-m^{\prime}\right|>j^{\beta} / 10$.

Then

$$
\text { mes } \Omega_{N}^{\prime} \leq e^{-N} \text {. }
$$

Proof. We cover $\tilde{\Lambda}$ with $\Lambda_{*}$ of the form (4.13). We use a resolvent expansion similar to the proof of Lemma 3.9 to obtain the opposite of (4.18). For that purpose we need to take away an additional set of $\omega$ so that $(3.42,3.43)$ hold on all $\Lambda_{*}$ of the form (4.13) at $\theta=0$.

For any $\Lambda_{*}(n, 2 j)$ such that $|n| \geq \exp \left[\frac{1}{2}(\log N)^{2}\right], \max (|n|,|2 j|) \asymp \exp \left[(\log N)^{2}\right]$, (4.14) is available. Take one such $\Lambda_{*}$ and let $\kappa=e^{-2 N} \gg e^{-N^{2}}$ for $N$ large. Assume (4.17) holds, so $\left|E-E_{k}\right| \leq e^{-N^{2}} \ll e^{-2 N}$ for some eigenvalue $E_{k}$ of $H_{\bar{N}}$. Lemma 4.3 then says that

$$
\left\|\left(E-H_{\Lambda_{*}}\right)^{-1}\right\| \leq e^{2 N}\left(1+2 e^{-N^{2}}\right)<2 e^{2 N}
$$

by taking away a set in $\omega$ of measure

$$
\leq C \kappa\left|\Lambda_{\bar{N}}\right|\left|\Lambda_{*}\right|
$$

Comparing (4.12) with (3.34) $J=N^{C}$ here. So

$$
\left\|\left(E-H_{\Lambda_{*}}\right)^{-1}\right\|<2 e^{2 N}<e^{J^{\beta^{\prime} / 2}}=e^{N^{C \beta^{\prime} / 2}},
$$

provided $C \beta^{\prime} / 2>1$ or $C>2 / \beta^{\prime}$. Therefore $(3.42,3.43)$ hold on this $\Lambda_{*}$.

Multiplying (4.20) by the number of all possible $\Lambda_{*}(n, 2 j)$ such that $|n| \geq \exp \left[\frac{1}{2}(\log N)^{2}\right]$, $\max (|n|,|2 j|) \asymp \exp \left[(\log N)^{2}\right]$, we have that $(3.42,3.43)$ are available on all such $\Lambda_{*}$ after taking away an additional set in $\omega$ of measure $\leq e^{-N}$.

For $\Lambda_{*}(n, 2 j)$ such that $|n|<\exp \left[\frac{1}{2}(\log N)^{2}\right]$, since $\max (|n|,|2 j|) \asymp \exp \left[(\log N)^{2}\right]$, $|j| \asymp \exp \left[(\log N)^{2}\right]$. So

$$
\operatorname{dist}\left(E, \sigma\left(H_{\Lambda_{*}}\right)\right) \asymp \exp \left[(\log N)^{2}\right], \quad \forall \omega \in[0,2 \pi)^{\nu},
$$

if $E$ satisfies (4.17). This is because $\left\|H_{\bar{N}}\right\| \leq \mathcal{O}(\bar{N})=N^{C} \ll \exp \left[(\log N)^{2}\right]$.

The proof now proceeds as the proof of Lemma 3.9 and we arrive at the conclusion. 


\section{Proof of the Theorem}

We use Lemme 4.1, 4.4 to prove that the Floquet Hamiltonian $K$ in (1.15), or rather its unitary equivalent $H$ in (1.19) has pure point spectrum. Let $\Omega_{N}, \Omega_{N}^{\prime}$ be the

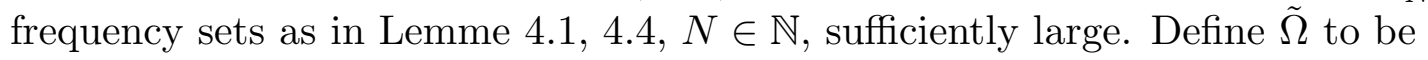

$$
\tilde{\Omega}=\mathbb{T}^{\nu} \backslash \bigcup_{N \geq N_{0}}\left(\Omega_{N} \cup \Omega_{N}^{\prime}\right), \quad N_{0} \gg 1 \text { (depending on } \delta \text { ), }
$$

then mes $\tilde{\Omega} \rightarrow(2 \pi)^{\nu}$ as $\delta \rightarrow 0$.

Fix $\omega \in \tilde{\Omega}$. From the Schnol-Simon theorem [CFKS, S], to prove $H$ in (1.19) has pure point spectrum, it suffices to prove that the generalized eigenfunctions have fast decay, hence are in $\ell^{2}$. More precisely, let $\psi$ be a non zero function on $\mathbb{Z}^{\nu+1}$ satisfying

$$
(H-E) \psi=0, \quad|\psi(m)| \leq 1+|m|^{c_{0}} \text { for all } m \in \mathbb{Z}^{\nu+1},
$$

where $E$ is arbitrary and $c_{0}>0$ is some constant. We will prove using Lemme 4.1, 4.4 that $\psi$ has subexponential decay and hence is in $\ell^{2}\left(\mathbb{Z}^{\nu+1}\right)$.

We first verify that (4.5) is satisfied. This implies that (4.17) is also satisfied as they are the same condition. So we need to show that there is some box $\Lambda_{\bar{N}}$ centered at $0, \Lambda_{\bar{N}}=[-\bar{N}, \bar{N}]^{\nu+1}$, for some $\bar{N} \asymp N^{C}\left(C>2 / \beta^{\prime}, 0<\beta^{\prime}<\min (1 / 20,1 / 5 \alpha)\right.$ as in Lemme 4.1,4.4), such that

$$
\left\|\left(H_{\bar{N}}-E\right)^{-1}\right\| \geq e^{N^{2}}
$$

For this we let

$$
T=\left\{(n, j) \in \Lambda_{\bar{N}} \mid j \in\left[-N^{C^{\prime}}, N^{C^{\prime}}\right]\right\},
$$

$C^{\prime}>1$ to be determined from (5.8-5.11), $C$ chosen to be $>C^{\prime}$. Let $\Lambda_{N C^{\prime}}$ be boxes of side length $2 N^{C^{\prime}}$. We cover $T$ with $\Lambda_{N^{C^{\prime}}}, \Lambda_{\bar{N}} \backslash T$ with $\Lambda_{*}$ of the form (4.13).

From Proposition 3.10, Lemma 3.7, there are at most $\bar{N}^{1-\tau_{0}} \quad\left(\tau_{0}>0\right)$ pairwise disjoint bad $\Lambda_{N C^{\prime}}$ boxes in $T$ by taking $C$ large enough. In $\Lambda_{\bar{N}} \backslash T$, there is at most 1 pairwise disjoint bad $\Lambda_{*}$ box by a further reduction in the $\tilde{\Omega}$ set as follows.

Assume $\exists(n, j) \in \Lambda_{*}, \exists(m, k) \in \Lambda_{*}^{\prime}, \Lambda_{*} \cap \Lambda_{*}^{\prime}=\emptyset$ such that

$$
\left|\lambda_{n, j}(\omega)-\lambda_{m, k}^{\prime}(\omega)\right| \leq \kappa,
$$

where $\lambda_{n, j}, \lambda_{m, k}^{\prime}$ are eigenvalues of $H_{\Lambda_{*}}, H_{\Lambda_{*}^{\prime}}$. From Proposition 3.3

$$
\begin{aligned}
\lambda_{n, j} & =n \cdot \omega+j+\mathcal{O}\left(j^{-1 / 4}\right) \\
\lambda_{m, k}^{\prime} & =m \cdot \omega+k+\mathcal{O}\left(k^{-1 / 4}\right) .
\end{aligned}
$$

So

$$
\left|\lambda_{n, j}-\lambda_{m, k}^{\prime}\right| \geq 1 / 2
$$

if $n=m$, since $(n, j) \neq(m, k)$. 
We only need to look at the case $n \neq m$. This is similar to the proof of Lemma 4.3, except we look at the first order eigenvalue variation in the $(n-m)$ direction. Let

$$
\phi_{n, j}=\sum_{\left(n^{\prime}, j^{\prime}\right) \in \Lambda_{*}} a_{n^{\prime}, j^{\prime}} \delta_{n^{\prime}, j^{\prime}}
$$

be the eigenfunction with eigenvalue $\lambda_{n, j}$ :

$$
H_{\Lambda_{*}} \phi_{n, j}=\lambda_{n, j} \phi_{n, j}
$$

and

$$
\psi_{m, k}=\sum_{\left(m^{\prime}, k^{\prime}\right) \in \Lambda_{*}^{\prime}} b_{m^{\prime}, k^{\prime}} \delta_{m^{\prime}, k^{\prime}}
$$

be the eigenfunction with eigenvalue $\lambda_{m, k}^{\prime}$ :

$$
H_{\Lambda_{*}^{\prime}} \psi_{m, k}=\lambda_{m, k} \psi_{m, k}
$$

We have for the first order variation:

$$
\begin{aligned}
& (n-m) \cdot \frac{\partial}{\partial \omega}\left[\lambda_{n, j}-\lambda_{m, k}^{\prime}\right] \\
= & (n-m) \cdot \frac{\partial}{\partial \omega}\left[\sum_{\left(n^{\prime}, j^{\prime}\right) \in \Lambda_{*}}\left|a_{n^{\prime}, j^{\prime}}\right|^{2} n^{\prime} \cdot \omega-\sum_{\left(m^{\prime}, k^{\prime}\right) \in \Lambda_{*}^{\prime}}\left|b_{m^{\prime}, k^{\prime}}\right|^{2} m^{\prime} \cdot \omega\right] \\
= & (n-m) \cdot \frac{\partial}{\partial \omega}\left[(n-m) \cdot \omega+\sum_{\left(n^{\prime}, j^{\prime}\right) \in \Lambda_{*}, n \neq n^{\prime}}\left|a_{n^{\prime}, j^{\prime}}\right|^{2}\left(n^{\prime}-n\right) \cdot \omega\right. \\
& \left.-\sum_{\left(m^{\prime}, k^{\prime}\right) \in \Lambda_{*}^{\prime}, m \neq m^{\prime}}\left|b_{m^{\prime}, k^{\prime}}\right|^{2}\left(m^{\prime}-m\right) \cdot \omega\right] \\
= & |n-m|^{2}+\mathcal{O}\left(\frac{1}{j^{1 / 20}}\right) \cdot j^{\beta^{\prime}}|n-m|+\mathcal{O}\left(\frac{1}{k^{1 / 20}}\right) k^{\beta^{\prime}}|n-m| \\
> & \frac{1}{2}|n-m|^{2} \\
\geq & \frac{1}{2},
\end{aligned}
$$

where we used (3.27) of Proposition 3.3 and the definitions of $\Lambda_{*}, \Lambda_{*}^{\prime}$ in (4.13). So

$$
\text { mes }\left\{\omega \| \lambda_{n, j}(\omega)-\lambda_{m, k}^{\prime}(\omega) \mid \leq \kappa, \omega \text { satisfies }(3.23)\right\} \leq C \kappa \text {. }
$$

Take $\kappa=e^{-(\log N)^{2}}$ and denote the set $\Omega_{N}^{\prime \prime}$ such that $\exists \Lambda_{*} \subset \Lambda_{\bar{N}}, \Lambda_{*}^{\prime} \subset \Lambda_{\bar{N}}, \Lambda_{*} \cap \Lambda_{*}^{\prime}=$ $\emptyset, \exists(n, j) \in \Lambda_{*}, \exists(m, k) \in \Lambda_{*}^{\prime}$, such that (5.4) holds. Then

$$
\operatorname{mes} \Omega_{N}^{\prime \prime} \leq e^{-\frac{1}{2}(\log N)^{2}},
$$

provided $2 / \beta^{\prime}<C \ll \log N$. 
Let

$$
\Omega \stackrel{\text { def }}{=} \tilde{\Omega} \backslash \bigcup_{N \geq N_{0}} \Omega_{N}^{\prime \prime},
$$

mes $\Omega \rightarrow(2 \pi)^{\nu}$ as $\delta \rightarrow 0$. We now assume $\omega \in \Omega$. Then in $\Lambda_{\bar{N}}, \bar{N} \asymp N^{C}$, there are at most $\bar{N}^{1-\tau_{0}}\left(0<\tau_{0}<1\right)$ bad $\Lambda_{N^{C^{\prime}}}$ boxes with centers in $T$ and 1 bad $\Lambda_{*}$ box with center in $\Lambda_{\bar{N}} \backslash T$. Since

$$
\frac{\bar{N}}{N^{5(\nu+1) C^{\prime}}}>\bar{N}^{\tau_{0}}
$$

for $C$ large enough, there has to be an annulus $A$ at a distance $\asymp N^{C}$ to the origin of thickness $10 N^{C^{\prime}}$ in $T$ and of thickness $N^{C} / 4$ in $\Lambda_{\bar{N}} \backslash T$ (as there is at most 1 bad $\Lambda_{*}$ box) devoid of bad points.

Since $(H-E) \psi=0$ from $(5.2)$, let

$$
\left(H_{\tilde{\Lambda}(c)}-E\right) \psi=\xi_{\tilde{\Lambda}}
$$

where $\tilde{\Lambda}(c)$ is a box of type $\Lambda=\Lambda_{N C^{\prime}}$ or $\Lambda_{*}$ centered in $c$. In view of $(1.20,5.2)$, the restriction on $\beta$ for $\Lambda_{*}$ in $(3.36)$

$$
\left|\xi_{\tilde{\Lambda}}(m)\right| \ll|m|^{c_{0}} e^{-c^{\prime}[\operatorname{dist}(m, \partial \tilde{\Lambda})]^{1 / 2}}
$$

if $\operatorname{dist}\left(m, \partial \tilde{\Lambda}^{(1)}\right)>|m-c|_{1}$, where

$$
\partial \tilde{\Lambda}^{(1)}=\left\{y \in \tilde{\Lambda}\left|\exists z \in \mathbb{Z}^{d} \backslash \tilde{\Lambda},\right| z-\left.y\right|_{1}=1\right\}
$$

and $\|_{1}$ is as defined above (3.46).

Let $c \in A, \tilde{\Lambda}(c) \subset A$. So $\tilde{\Lambda}(c)$ is a good box of type $\Lambda_{N C^{\prime}}$ with centers in $T$ or $\Lambda_{*}$ of the form (4.13) with centers in $\Lambda \backslash T$. Estimates $(3.42,3.80)$ are available. From (5.9)

$$
\psi=\left(H_{\tilde{\Lambda}(c)}-E\right)^{-1} \xi_{\tilde{\Lambda}}
$$

Using (5.10), (3.42) or (3.80), we have that

$$
|\psi(c)|<e^{-N^{2}}
$$

provided $C^{\prime}>2 / \beta^{\prime}$ for all $c \in A$, such that $\exists \tilde{\Lambda}(c) \subset A$. We now choose $2 / \beta^{\prime}<C^{\prime}<$ $C \ll \log N$, so that (5.8) is satisfied. (5.11) implies immediately that

$$
|\psi(m)|<e^{-N^{2}}
$$

for all $m \in A^{\prime} \subset A$, where $A^{\prime}$ is a smaller annulus contained in $A$ of thickness $6 N^{C^{\prime}}$ in $T$ and thickness $N^{C} / 5$ in $\Lambda_{\bar{N}} \backslash T$.

Since dist $A^{\prime}$ to the origin $\asymp N^{C}$, one can always find a square $\Lambda_{\bar{N}}$ centered at the origin, $\bar{N} \asymp N^{C}$, such that $\partial \Lambda_{\bar{N}} \subset A^{\prime}$,

$$
\begin{aligned}
& \operatorname{dist}\left(\partial \Lambda_{\bar{N}}, A^{\prime} \cap T\right)>2 N^{C^{\prime}}, \\
& \operatorname{dist}\left(\partial \Lambda_{\bar{N}}, A^{\prime} \cap T^{c}\right)>N^{C} / 10 .
\end{aligned}
$$


Using (5.9) with $\Lambda_{\bar{N}}$ in place of $\tilde{\Lambda}(c)$, we have

$$
\psi=\left(H_{\bar{N}}-E\right)^{-1} \xi_{\bar{N}}
$$

Using (5.12-5.14), we have $\left\|\xi_{\bar{N}}\right\|<e^{-N^{2}}$ by slightly increasing $C^{\prime}$ if necessary. Since we may always assume $\psi(0)=1,(5.10,5.11)$ give that

$$
\left\|\left(H_{\bar{N}}-E\right)^{-1}\right\|>e^{N^{2}}
$$

Subexponential decay of eigenfunctions of $H$.

Let $K=\exp \left[(\log N)^{2}\right]$. Lemme 4.1, 4.4 then imply (via a proof similar to the proofs of Lemme 3.8 and 3.9) that the Green's function of the set

$$
U \stackrel{\text { def }}{=} \Lambda_{2 K}(0) \backslash \Lambda_{K}(0)
$$

where $\Lambda_{L}(0)=[-L, L]^{\nu+1}$, exhibits off-diagonal decay, i.e.,

$$
\left|G_{U}(E, m, n)\right| \leq e^{-|m-n|^{1 / 4}} \quad m, n \in U, \quad|m-n|>K / 10
$$

Let $\xi_{U}$ be defined as in (5.9) with $U$ replacing $\tilde{\Lambda}$. Fix $m \in U$. Assume dist $(m, \partial U) \geq$ $K / 4$. For $n$ such that dist $(n, \partial U)>K / 10$, we use the bound in (5.10) with $\xi_{U}$ replacing $\xi_{\tilde{\Lambda}}$. Otherwise we use the fact that $\xi_{U}$ is polynomially bounded since $\psi$ is polynomially bounded. The equality

$$
\psi(m)=\sum_{n} G_{U}(E, m, n) \xi(n)
$$

then implies that

$$
|\psi(m)| \leq e^{-|m|^{\kappa}} \quad(0<\kappa<1 / 4),
$$

provide $K$ and thus $|m|$ is large enough. 


\section{REFERENCES}

[Ba] S. Basu, On bounding the Betti numbers and computing the Euler characteristic of semialgebraic sets, Discrete Comput. Geom. 22 (1999), 1-18.

[Be] J. Bellissard, Stochastic Process in Classical and Quantum Systems, Springer, Berlin, 1986.

[B1] J. Bourgain, Construction of quasi-periodic solutions for Hamiltonian perturbations of linear equations and applications to nonlinear PDE, IMRN 11 (1994), 475-497.

[B2] J. Bourgain, Quasi-periodic solutions of Hamiltonian perturbations of 2D linear Schrödinger equations, Ann. Math 148 (1998), 363-439.

[B3] J. Bourgain, Green's function estimates for lattice Schrödinger operators and applications, Princeton University Press, 2005.

[BGS] J. Bourgain, M. Goldstein, W. Schlag, Anderson localization for Schrödinger operators on $\mathbb{Z}^{2}$ with quasi-periodic potential, Acta Math. 188 (2002), 41-86.

[BW1] J. Bourgain, W.-M. Wang, Anderson localization for time quasi-periodic random Schrödinger and wave equations, Commun. Math. Phys. 248 (2004), 429-466.

[BW2] J. Bourgain, W.-M. Wang, Quasi-periodic solutions for nonlinear random Schrödinger, J. European Math. Society (to appear).

[BBL] A. Boutel de Monvel-Berthier, L. Boutet de Monvel, G. Lebeau, Sur les valeurs propres d'un oscillateur harmonique perturbe, J. d'Anal. Math. 58 (1992).

[Co1] M. Combescure, A quantum particle in a quadrupole radio-frequency trap, Ann. Inst. Henri. Poincare 44 (1986), 293-314.

[Co2] M. Combescure, The quantum stability problem for time-periodic perturbation of the harmonic oscillator, Ann. Inst. Henri. Poincare 47 (1987), 63-83, 451-454.

[CW] W. Craig, C. E. Wayne, Newton's method and periodic solutions of nonlinear wave equation, Commun. Pure Appl. Math. 46 (1993), 1409-1498.

[CFKS] H. L. Cycon, R. G. Froese, W. Kirsch, B. Simon, Schrödinger Operators, Springer-Verlag, 1987.

[DS] P. Duclos, P. Stovicek, Floquet Hamiltonians with pure point spectrum, Commun. Math. Phys. 177 (1996), 327-347.

[E] L. H. Eliasson, Almost reducibility of linear quasi-periodic systems, Proceedings of Symp in Pure Math 69 (2001), 679-705.

[EK] L. H. Eliasson, S. B. Kuksin, KAM for the nonlinear Schrödinger equation (2006).

[EV] V. Enss, K. Veselic, Bound states and propagating states for time-dependent Hamiltonians, Ann IHP 39 (1983), No. 2, 159-191.

[FS] J. Fröhlich, T. Spencer, Absence of diffusion in the Anderson tight binding model for large disorder, Commun. Math. Phys. 88 (1983), 151-184.

[GB] F. Germinet, S. de Bievre,, Dynamical localization for discrete and continuous random Schrödinger operators, Commun. Math. Phys. 194 (1998), 323-341.

[GK] F. Germinet, A. Klein, Bootstrap multiscale analysis and localization in random media, Commun. Math. Phys. 222 (2001), 415-448.

[GR] I.S. Gradshteyn, I. M. Ryzhik, Table of Integrals, Series and Products (6th ed), Academic Press, 2000.

[GY] S. Graffi, K. Yajima, Absolute continuity of the Floquet spectrum for a nonlinearly forced harmonic oscillator, Commun. Math. Phys. 215 (2000), 245-250. 
[HLS] G. Hagedorn, M. Loss, J. Slawny, Non stochasticity of time-dependent quadratic Hamiltonians and the spectra of canonical transformations, J. Phys. A (1986), 521-531.

[Ho] J. S. Howland, Stationary scattering theory for time-dependent Hamiltonians, Math. Ann. 207 (1974), 315-335.

[JL] H. R. Jauslin, J. L. Lebowitz, Spectral and stability aspects of quantum chaos, Chaos 1 (1991), 114-121.

[KRY] L. Kapitanski, I. Rodnianski, K. Yajima, On the fundamental solution of a perturbed harmonic oscillator, Topo. Methods Nonlinear Anal. 9 (1997).

[Ka] T. Kato, Perturbation Theory for Linear Operators, Springer-Verlag, Berlin-Heidelberg, New York, 1980.

[Ku1] S. Kuksin, Hamiltonian perturbation of infinite-dimensional linear systems, Funts. Anal. Prilozh. 21, 149-179; English translation in Funct. Anal. Appl. 21 (1987), 192-205.

[Ku2] S. Kuksin, Nearly Integrable Infinite-Dimensional Hamiltonian Systems, Lect. Notes. Math. 1556, Springer-Verlag, 1993.

[KP] S. Kuksin, J. Pöschel, Invariant Cantor manifolds of quasi-periodic osillations for a nonlinear Schrödinger equation, Ann. Math. 143 (1996), 149-179.

[PBM] A. P. Prudnikov, Yu. A. Brychkov, O. I. Marichev, Integrals and Series, Vol I, Gordon and Breach Scien Publishers, 1986.

[S] I. Schnol, On the behaviour of the Schrödinger equation, Mat. Sb. (Russian) (1957), 273286.

[T] S. Thangavelu, Lectures on Hermite and Laguerre Expansions, Math. Notes 42, Princeton University Press, 1993.

[W] W.-M. Wang, Quasi-periodic solutions of nonlinearly perturbed quantum harmonic oscillator (in preparation).

[Y1] K. Yajima, Resonances for the AC-Stark effect, Commun. Math. Phys. 78 (1982), 331-352.

[Y2] K. Yajima, On smoothing property of Schrödinger propagators, Lect. Notes Math. 1450 (1989), 20-35.

[Y3] K. Yajima, Schrödinger evolution equations with magnetic fields, J. d'Anal. Math. 56 (1991), 29-76.

[YK] K. Yajima, H. Kitada, Bound states and scattering states for time periodic Hamiltonians, Ann. IHP, A 39 (1983), 145-157.

[Z] S. Zelditch, Reconstruction of singularities for solutions of Schrödinger's equation, Commun. Math. Phys. 90 (1983), 1-26.

Departement de Mathematique, Universite Paris Sud, 91405 Orsay Cedex, France; Department of Mathematics, University of Massachussetts, Amherst, Ma 01003, USA

E-mail address: wei-min.wang@math.u-psud.fr, weimin@math.umass.edu 